\title{
1 An Integrative Analysis of the Age-Associated Genomic, Transcriptomic and Epigenetic
}

\section{Landscape across Cancers}

3 Kasit Chatsirisupachai ${ }^{1}$, Tom Lesluyes ${ }^{2}$, Luminita Paraoan ${ }^{3}$, Peter Van Loo $^{2}$, João Pedro de

$4 \quad$ Magalhães $^{1 *}$

$5{ }^{1}$ Integrative Genomics of Ageing Group, Institute of Life Course and Medical Sciences,

$6 \quad$ University of Liverpool, Liverpool L7 8TX, UK.

$7 \quad{ }^{2}$ The Francis Crick Institute, London NW1 1AT, UK.

$8{ }^{3}$ Department of Eye and Vision Science, Institute of Life Course and Medical Sciences,

$9 \quad$ University of Liverpool, Liverpool L7 8TX, UK.

$10 *$ email: jp@senescence.info

\section{Abstract}

Age is the most important risk factor for cancer, as cancer incidence and mortality increase with age. However, how molecular alterations in tumours differ among patients of different age remains largely unexplored. Here, using data from The Cancer Genome Atlas, we comprehensively characterised genomic, transcriptomic and epigenetic alterations in relation to patients' age across cancer types. We showed that tumours from older patients present an

17 overall increase in genomic instability, somatic copy-number alterations (SCNAs) and somatic mutations. Age-associated SCNAs and mutations were identified in several cancer-driver genes across different cancer types. The largest age-related genomic differences were found in gliomas and endometrial cancer. We identified age-related global transcriptomic changes and demonstrated that these genes are controlled by age-associated DNA methylation changes. This study provides a comprehensive view of age-associated alterations in cancer and underscores age as an important factor to consider in cancer research and clinical practice.

25 Keywords: ageing, carcinoma, brain cancer, geriatric oncology, single nucleotide variants 
Introduction

Age is the biggest risk factor for cancer, as cancer incident and mortality rates increase exponentially with age in most cancer types ${ }^{1}$. However, the relationship between ageing and molecular determinants of cancer remains to be characterised. Cancer arises through the interplay between somatic mutations and selection, in a Darwinian-like process ${ }^{2,3}$. Thus, apart

31 from the mutation accumulation with age ${ }^{4-6}$, microenvironment changes during ageing could

32 also play a role in carcinogenesis ${ }^{2,7,8}$. We therefore hypothesise that, due to the differences in selective pressures from tissue environmental changes with age, tumours arise from patients across different ages might harbour different molecular landscapes; consequently, some molecular changes might be more or less common in older or younger patients.

37 genome in relation to clinical factors, including gender ${ }^{9,10}$ and race $^{11,12}$. These studies

demonstrated gender- and race-specific biomarkers, actionable target genes and provided clues

to understanding the biology behind the disparities in cancer incidence, aggressiveness and treatment outcome across patients from different backgrounds. Although the genomic alterations in childhood cancers and the differences with adult cancers have been systematically

42 characterised $^{13,14}$, the age-related genomic landscape across adult cancers remains elusive.

43 Specific age-associated molecular landscapes have been reported in the cancer genome of

44 several cancer types, for example, glioblastoma ${ }^{15}$, prostate cancer ${ }^{16}$ and breast cancer ${ }^{17}$.

45 However, these studies focused mainly on a single cancer type and only on some molecular 46 data types.

47 Here, using data from The Cancer Genome Atlas (TCGA), we systematically 48 investigated age-related differences in genomic instability, somatic copy number alterations 49 (SCNAs), somatic mutations, pathway alterations, gene expression, and DNA methylation 50 landscape across various cancer types. We show that, in general, genomic instability and 
51 mutations frequency increase with age. We identify several age-associated genomic alterations

52 in cancers, particularly in low-grade glioma and endometrial carcinoma. Moreover, we also

53 demonstrate that age-related gene expression changes are controlled by age-related DNA

54 methylation changes and that these changes are linked to numerous biological processes.

55

56

57

58

59

60

61

62

63

64

65

66

67

68

69

70

71

72

73

74

75 


\section{Results}

77 Association between age and genomic instability, loss of heterozygosity, and wholegenome duplication

To gain insight into the role of patient age into the somatic genetic profile of tumours, we evaluated associations between patient age and genomic features of tumours in TCGA data (Table 1, Supplementary Table 1). Using multiple linear regression adjusting for gender, race, and cancer type, we found that genomic instability (GI) scores increase with age in pan-cancer data $\left(\right.$ adj. $R$-squared $=0.35$, p-value $\left.=5.98 \times 10^{-7}\right)($ Fig. 1a $)$. We next applied simple linear regression to investigate the relationship between GI scores and age for each cancer type. Cancer types with a significant association (adj. p-value $<0.05$ ) were further adjusted for clinical variables. We found a significant positive association between age and GI score in seven cancer types (adj. p-value < 0.05) (Fig. 1b, Supplementary Fig. 1a and Supplementary

Table 2). Cancer types with the strongest significant positive association were low-grade glioma, ovarian cancer, endometrial cancer, and sarcoma. This result indicates that the level of genomic instability increases with the age of cancer patients in several cancer types. allele, causing an allelic imbalance, and priming the cell for another defect at the other remaining allele of the respective genes ${ }^{18}$. To investigate whether there is an association between patients' age and $\mathrm{LOH}$, we quantified percent genomic LOH. By using simple linear regression, we found a significant positive association between age and pan-cancer percent genomic $\mathrm{LOH}\left(\mathrm{p}\right.$-value $\left.=1.20 \times 10^{-21}\right)$. However, this association was no longer significant in

97 a multiple linear regression analysis (adj. R-squared $=0.32$, p-value $=0.289)$ (Fig. $1 \mathrm{c})$. Thus,

98 it is likely that this association might be cancer type-specific. We then performed a linear 99 regression between age and percent genomic LOH for each cancer type. Six cancer types 100 showed a positive association between age and percent genomic LOH (adj. p-value $<0.05$ ) 
101 (Fig. 1d, Supplementary Fig. 1b, and Supplementary Table 3). The strongest positive

102

103

104

105

106

107

108

109

110

111

112

113

114

115

116

117

118

119

120

121

122

123

124

125

associations were found in low-grade glioma and endometrial cancer (adj. p-value $<0.05$ ), corroborate with the increase in GI score with age. On the other hand, lung adenocarcinoma, oesophageal and liver cancer demonstrated a negative correlation between percent genomic LOH and age (adj. p-value $<0.05)$.

Whole-genome duplication (WGD) is important in increasing the adaptive potential of the tumour and has been linked with a poor prognosis ${ }^{19-21}$. We investigated the relationship between age and WGD using logistic regression. For the pan-cancer analysis, we found an increase in the probability that WGD occurs with age, using multiple logistic regression accounting for gender, race, and cancer type (odds ratio per year $(\mathrm{OR})=1.0066,95 \%$ confidence interval $(\mathrm{CI})=1.0030-1.0103, \mathrm{p}$-value $\left.=3.84 \times 10^{-4}\right)($ Fig. 1e). For the cancerspecific analysis, a significant positive association was found in ovarian and endometrial cancer (adj. p-value $<0.05, \mathrm{OR}=1.0320$ and $1.0248,95 \% \mathrm{CI}=1.0151-1.0496$ and $1.0024-$ 1.0483, respectively) (Fig. 1e and Supplementary Table 4), indicating that tumours from older patients are more likely to have doubled their genome. Taken together, the findings indicate that tumours from patients with an increased age tend to harbour a more unstable genome and a higher level of LOH in several cancer types. Notably, the strongest association between age and an increase in genome instability, LOH, and WGD was evident in endometrial cancer, suggesting the potential disparities in cancer genome landscape with age in this cancer type.

\section{Age-associated somatic copy-number alterations}

We used GISTIC2.0 to identify recurrently altered focal- and arm-level SCNAs ${ }^{22}$. We calculated the SCNA score, as a representation of the level of SCNA occurring in a tumour ${ }^{12,23}$. For each tumour, the SCNA score was calculated at three different levels: focal-, arm- and chromosome-level, and the overall score calculated from the sum of all three levels. We used 
simple linear regression to identify the association between age and overall SCNA scores.

127 Cancer types that displayed a significant association were further adjusted for clinical

128 variables. Consistent with the GI score results described above, the strongest positive

129 association between age and overall SCNA scores was found in low-grade glioma, ovarian and

130 endometrial cancers. Other cancer types for which a positive association between age and overall SCNA score was observed were thyroid cancer and clear cell renal cell carcinoma (adj. p-value $<0.05)$. On the other hand, lung adenocarcinoma is the only cancer type exhibiting a negative association between overall SCNA score and age (Fig. 2a, Supplementary Fig. 2a, and Supplementary Table 5). The different SCNA classes (focal- and chromosome/arm-level) may arise through different biological mechanisms ${ }^{12,21}$, therefore we separately analysed the association between age and focal- and chromosome/arm-level SCNA scores. Most cancers that showed a significant relationship between age and overall SCNA score also had an association between age and both chromosome/arm-level and focal-level SCNA scores (Fig. 2b-c, Supplementary Fig. 2b-c, and Supplementary Table 5). The only exception was in cervical cancer, with a significant association between age and chromosome/arm-level but not with focal-level and overall SCNA scores.

We next identified the chromosomal arms that tend to be gained and lost more often

143 with age, for 25 cancer types with sufficient samples (at least 100 tumours, Table 1). We

144 conducted the logistic regression on the significant recurrently gained and lost arms that were 145 identified by GISTIC2.0 for each cancer type. The significant association between age and 146 chromosomal arm gains and losses are shown in Fig. Fig. 2d, e, respectively (adj. p-value < 147 0.05) (Supplementary Fig. 3, Supplementary Table 6). The gain of chromosome 7p, 7q, 20p, 148 and 20q significantly increased with age in several cancer types including two types of gliomas,

149 low-grade glioma and glioblastoma. On the other hand, the gain of chromosome 10p decreased 150 with increased age in gliomas (Fig. 2d and 2f). For the arm losses, there was an increased 
occurrence of loss in 11 arms with advanced age in endometrial cancer (Fig. 2e and 2g), association between age and SCNA scores, also exhibited a significant increase or decrease in losses with age in multiple arms (Fig. 2e-f, Supplementary Fig. 3). We also observed that the losses of chromosome 10p and 10q increased with age in gliomas. Recurrent losses of

IDH-mutated gliomas were predominantly found in younger patients. number of significant regions was found in endometrial cancer (23 gain and 25 loss regions), followed by ovarian cancer (13 gain 2 loss regions) and low-grade glioma (9 gain and 5 loss regions) (Fig. 3b-c, Supplementary Fig. 4). the SCNA level and gene expression for tumours that have both types of data using Pearson correlation. In total, 81 genes in the list of previously identified cancer driver genes

171 (Supplementary Table 8) were presented in at least one significant age-associated focal region in at least one cancer type and showed a significant correlation between SCNAs and gene expression (adj. p-value < 0.05) (Fig. 3d). For example, regions showing an increased gain

174 with age in endometrial cancer included 1q22, where the gene RIT1 is located in $(\mathrm{OR}=1.0355$, $17595 \% \mathrm{CI}=1.0151-1.0571$, adj. p-value $=0.0018)($ Fig. 3c, e). The Ras-related GTPases RIT1 
gene has been reported to be highly amplified and correlated with poor survival in endometrial

177 cancer ${ }^{25}$. Therefore, an increase in the gain of the RIT1 gene with age might relate to a poor

178 prognosis in older patients. The 16 p13.3 loss increased in older endometrial cancer patients

$179(\mathrm{OR}=1.0335,95 \% \mathrm{CI}=1.0048-1.0640$, adj. $\mathrm{p}$-value $=0.0328)$. This region contains the $\mathrm{p} 53$ coactivator gene $C R E B B P$. The gain of $8 \mathrm{q} 24.21$ harbouring the oncogene $M Y C$ decreased with patient age in low-grade glioma $(\mathrm{OR}=0.9737,95 \% \mathrm{CI}=0.9541-0.9927$, adj. $\mathrm{p}$-value $=0.0128)$ and ovarian cancer $(\mathrm{OR}=0.9729,95 \% \mathrm{CI}=0.9553-0.9904$, adj. $\mathrm{p}$-value $=0.0063)($ Fig. $3 \mathrm{~d}, \mathrm{e})$. In addition, in low-grade glioma, we found an increase in 9p21.3 loss with age $(\mathrm{OR}=1.0332$, $95 \% \mathrm{CI}=1.0174-1.0496$, adj. $\mathrm{p}$-value $=0.00017)$. This region contains the cell cycle-regulator genes $C N K N 2 A$ and $C D K N 2 B$ (Fig. 3b, d, e). The full list of age-associated focal regions across cancer types and the correlation between SCNA status and gene expression can be found in and SCNAs level across cancer types. We also identified age-associated arms and focalregions, and these regions harboured several cancer-driver genes. Our results suggest a possible contribution of different SCNA events in cancer initiation and progression of patients with different ages.

\section{Age-associated somatic mutations in cancer}

The increase in the mutational burden with age is well-established ${ }^{4-6}$. This age-related mutation accumulation is largely explained by a clock-like mutational process, the spontaneous deamination of 5-methylcytosine to thymine ${ }^{5}$. As expected, we confirmed the correlation between age and mutation load (somatic non-silent SNVs and indels) in the pan-cancer cohort using multiple linear regression adjusting for gender, race, and cancer type (adj. R-squared =

1990.53, p-value $\left.=1.41 \times 10^{-37}\right)($ Supplementary Fig. 5a). For cancer-specific analysis, 18 cancer 
201 (adj. p-value < 0.05) (Supplementary Fig. 5, Supplementary Table 9). Only endometrial cancer

202 showed a negative correlation between mutational burden and age. We observed a high 203 proportion of hypermutated tumours ( $>1,000$ non-silent mutations per exome) from younger 204 endometrial cancer patients. Thirteen out of 38 tumours (34\%) from the younger patients (age $205 \leq 50)$ were hypermutated tumours, while there were only 42 hypermutated tumours from 383 tumours from older patients $(11 \%)($ Fisher's exact, $p$-value $=0.0003)($ Fig. $4 a)$. Microsatellite instability (MSI) is a unique molecular alteration caused by defects in DNA mismatch repair ${ }^{26,27}$. The MSI-high (MSI-H) tumours occur as a subset of high mutational burden tumours $^{28}$. We investigated whether high mutation loads in endometrial cancer from young patients were due to the presence of MSI-H tumours. Using multiple logistic regression, we found that MSI-H tumours were associated with younger endometrial cancer $(\mathrm{OR}=0.9751$, $95 \% \mathrm{CI}=0.9531-0.9971, \mathrm{p}$-value $=0.0264)($ Fig. $4 \mathrm{~b})$. Another source of hypermutation in cancer is the defective DNA polymerase proofreading ability by mutations in polymerase $\varepsilon$

$214(P O L E)$ or polymerase $\delta(P O L D 1)$ genes $^{29,30}$. We showed that mutations in POLE $(\mathrm{OR}=$ $0.9690,95 \% \mathrm{CI}=0.9422-0.9959, \mathrm{p}$-value $=0.0243)$ and $P O L D 1(\mathrm{OR}=0.9573,95 \% \mathrm{CI}=$ 0.9223-0.9925, $\mathrm{p}$-value $=0.0177)$ were both more prevalent in younger endometrial cancer

217 patients (Fig. 4c). Therefore, the negative correlation between age and mutation loads in endometrial cancer could be explained by the presence of hypermutated tumours in younger

219 patients, which are associated with MSI-H and POLE/POLD1 mutations. Previous studies on

$220 P O L E$ and MSI-H subtypes in hypermutated endometrial tumours revealed that these subtypes associated with a better prognosis when compared with the copy-number high subtype ${ }^{31-33}$.

222 Together with our SCNA results, younger UCEC patients are likely to associate with a POLE and MSI-H subtypes, high mutation rate and better survival, whilst tumours from older patients

224 are characterized by high SCNAs and are generally associated with a worse prognosis. We extended the age and MSI-H analysis to other cancer types known to have a high prevalence 
of MSI-H tumours, including colon, rectal, and stomach cancers ${ }^{26}$. Only in stomach cancer we

227 found an association between older age and the presence of MSI-H tumours (OR = 1.0392, 95\%CI $=1.0091-1.0720$, p-value $=0.01$, Supplementary Fig. 6a). When we further examined the association between age and mutations in POLE and POLD1 in other cancers apart from endometrial cancer, no significant association was observed (Supplementary Fig. 6b). understand this, we conducted logistic regression to investigate genes that are more or less likely to be mutated with an increased age. To prevent the potential bias caused by hypermutated tumours, we restricted the analysis to samples with $<1,000$ non-silent mutations per exome (Table 1). We first investigated the association between age and pan-cancer genelevel mutations. Using multiple logistic regression correcting for gender, race, and cancer type, mutations in $I D H 1\left(\mathrm{OR}=0.9619,95 \% \mathrm{CI}=0.9510-0.9730\right.$, adj. p-value $\left.=4.18 \times 10^{-10}\right)$ and $\operatorname{ATR} X\left(\mathrm{OR}=0.9803,95 \% \mathrm{CI}=0.9724-0.9881\right.$, adj. $\mathrm{p}$-value $\left.=9.85 \times 10^{-6}\right)$ showed a negative association with age. On the other hand, mutations in PIK3CA were more common in older individuals $\left(\mathrm{OR}=1.0082,95 \% \mathrm{CI}=1.0022-1.0143\right.$, adj. p-value $\left.=4.18 \times 10^{-10}\right)($ Fig. $4 \mathrm{~d}) . \mathrm{We}$ next identified genes in which mutations associated with age in a cancer-specific manner in 24 cancers with at least 100 samples (Table 1). Using logistic regression, we identified 35

244 mutations from 13 cancers that increased or decreased with the patients' age (adj. p-value < 245 0.05) (Fig. 4e-f, Supplementary Fig. 7 and Supplementary Table 10). The most striking negative associations between mutations and age in low-grade glioma and glioblastoma were

247 found in $I D H 1(\mathrm{OR}=0.9509$ and $0.8962,95 \% \mathrm{CI}=0.9328-0.9686$ and $0.8598-0.9291$, adj. p248 value $=4.33 \times 10^{-7}$ and $1.88 \times 10^{-9}$, respectively $), \operatorname{ATR} X(\mathrm{OR}=0.9471$ and $0.9120,95 \% \mathrm{CI}=$ $249 \quad 0.9310-0.9628$ and $0.8913-0.9466$, adj. $\mathrm{p}$-value $=1.75 \times 10^{-10}$ and $2.45 \times 10^{-8}$, respectively), and 250 TP53 $(\mathrm{OR}=0.9431$ and $0.9736,95 \% \mathrm{CI}=0.9274-0.9582$ and $0.9564-0.9905$, adj. $\mathrm{p}$-value $=$ 
$1.13 \times 10^{-12}$ and , respectively). Our observation was consistent with the fact that the median age

IDH1 mutations often co-occurred with ATRX and TP53 mutations, and mutations in these three genes were more prevalent in gliomas without EGFR mutations ${ }^{15,35}$. Indeed, we found that EGFR mutations were more common in older low-grade glioma patients $(\mathrm{OR}=1.0865$, $95 \% \mathrm{CI}=1.0525-1.1258$, adj. $\mathrm{p}$-value $\left.=4.35 \times 10^{-7}\right)($ Fig. $4 \mathrm{f})$. Moreover, our SCNA analysis revealed an increase in the gain of $E G F R$ with age in low-grade glioma but not in glioblastoma (Fig. 3d), suggesting the difference in age-associated genomic landscape between the two glioma types. Together with the SCNA results, gliomas from younger patients are associated with IDH1, ATRX, and TP53 mutations, lower SCNAs, and longer survival. In contrast, gliomas from older patients were more likely to be IDH-WT with EGFR mutations, chromosome 7 gain and 10 loss, $C D K N 2 A$ deletion and worse prognosis. depending on cancer types. For instance, PTEN mutations decreased with patient's age in colon $(\mathrm{OR}=0.9347,95 \% \mathrm{CI}=0.8935-0.9738$, adj. $\mathrm{p}$-value $=0.0029)$ and endometrial cancers $(\mathrm{OR}$ $=0.9586,95 \% \mathrm{CI}=0.9331-0.9840$, adj. $\mathrm{p}$-value $=0.0033)$ but increased with age in cervical cancer $(\mathrm{OR}=1.0550,95 \% \mathrm{CI}=1.0174-1.0959$, adj. $\mathrm{p}$-value $=0.0067) . C D H 1$ mutations were more frequent in younger stomach cancer patients $(\mathrm{OR}=0.9414,95 \% \mathrm{CI}=0.9027-0.9800$, adj. $\mathrm{p}$-value $=0.0061)$ but more common in older breast cancer patients $(\mathrm{OR}=1.0218,95 \% \mathrm{CI}=$ 1.0049-1.0392, adj. p-value $=0.0171)$. These results highlight cancer-specific patterns of

272 genomic alterations in relation to age. Overall, our results demonstrate that non-silent 
mutation patterns. These patterns might point out age-associated disparities in carcinogenesis, molecular subtypes and survival outcome.

\section{7}

\section{Age-associated alterations in oncogenic signalling pathways}

As we have identified numerous age-associated alterations in cancer driver genes in

both SCNA and somatic mutation levels, we asked if the age-associated patterns also exist in

which had comprehensively characterized 10 highly altered signalling pathways in cancers ${ }^{36}$.

To make the subsequent analysis comparable to previous analyses, we restricted the analysis

to samples that were used in our previous analyses, yielding 8,055 samples across 33 cancer

types (Table 1). Using logistic regression adjusting for gender, race and cancer type, we

p-value $<0.05$ ), indicating that the genes in these pathways are altered more frequently in older

patients, concordant with the increase in overall mutations and SCNAs with age (Fig. 5a,

Supplementary Table 11). The strongest association was found in cell cycle $(\mathrm{OR}=1.0122$,

$95 \% \mathrm{CI}=1.0076-1.0168$, adj. $\mathrm{p}$-value $\left.=1.40 \times 10^{-6}\right)$ and $\mathrm{Wnt}$ signalling $(\mathrm{OR}=1.0122,95 \% \mathrm{CI}$

the cancer-specific association between age and oncogenic signalling alterations for cancer

types that contained at least 100 samples. In total, we identified 28 significant associations across 15 cancer types (adj. p-value < 0.05) (Fig. 5b, Supplementary Table 11). Alterations in

Hippo and TP53 signalling pathways significantly associated with age, both positively and 
300

301

302

303

304

305

306

307

308

in $C D K N 2 A$ and $C D K N 2 B$ deletions with age as well as epigenetic silencing of $C D K N 2 A$ in older patients (Fig. 5c). On the other hand, TP53 pathway alteration was more pronounced in younger patients $\left(\mathrm{OR}=0.9520,95 \% \mathrm{CI}=0.9372-0.9670\right.$, adj. $\mathrm{p}$-value $\left.=2.63 \times 10^{-8}\right)$, due to the mutations in the TP53 gene (Fig. 5c). In endometrial cancer, two pathways - Hippo (OR $=$ $0.9681,95 \% \mathrm{CI}=0.9459-0.9908$, adj. $\mathrm{p}$-value $=0.0126)$ and $\mathrm{Wnt}(\mathrm{OR}=0.9741,95 \% \mathrm{CI}=$ 0.9541-0.9946, adj. $\mathrm{p}$-value $=0.0240)$ - showed a negative association with age, that may be explained by the presence of hypermutated tumours in younger patients. Collectively, we reported pathway alterations in relation to age in several cancer types, highlighting differences in oncogenic pathways that might be important in cancer initiation and progression in an agerelated manner.

\section{Age-associated gene expression and DNA methylation changes}

Apart from the genomic differences with age, we investigated age-associated transcriptomic and epigenetic changes across cancers. We separately performed multiple linear regression analyses on gene expression data and methylation data of 24 cancer types that contained at least 100 samples in both types of data (Table 1). We noticed that, across all genes, the regression coefficient of age on gene expression negatively correlated with the regression coefficient of age on methylation in all cancer types (Supplementary Fig. 8), suggesting that the global changes of gene expression and methylation with age are in the opposite direction. This supports the established role of DNA methylation in suppressing gene expression. Numbers of significant differentially expressed genes with age (age-DEGs) (adj. p-value < 0.05, Supplementary Table 12) varied from nearly 5,000 up- and down-regulated genes in lowgrade glioma to no significant gene in 5 cancers. Similarly, we also identified significant differentially methylated genes with age (age-DMGs, Supplementary Table 13) (adj. p-value $<0.05$ ), the number of age-DEGs and age-DMGs were consistent for most cancer types (Fig. 
6a). We next focused our analysis on 10 cancer types that contained at least 150 age-DEGs and cancer, papillary renal cell carcinoma, ovarian cancer, liver cancer, acute myeloid leukaemia, melanoma, and prostate cancer. We identified overlapping genes between age-DEGs and ageDMGs and found that most of them, from $84 \%$ (37/44 genes) in ovarian cancer to $100 \%$ in acute myeloid leukaemia (57 genes) and prostate cancer ( 7 genes), were genes that presented increased methylation and decreased expression with age and genes that had decreased methylation and increased expression with age (Fig. 6b-c, Supplementary Fig. 9,

333 Supplementary Table 14). We further examined the correlation coefficient between methylation and expression comparing between 4 groups of genes 1) overlap genes between age-DMGs and age-DEGs (age-DMGs-DEGs), 2) age-DMGs only, 3) age-DEGs only, and 4) other genes. We found that age-DMGs-DEGs had the most negative correlation between DNA methylation and expression when comparing with other groups of genes (Fig. 6d,

338 Supplementary Fig. 10, Supplementary Table 15), highlighting that age-associated gene expression changes in cancer are repressed, at least in part, by DNA methylation.

341 into the expression and methylation changes with age. We identified various significantly

342 enriched Gene Ontology (GO) terms across cancers (Fig. 6e, Supplementary Fig. 11,

343 Supplementary Table 16). Notably, several GO terms were enriched in both expression and 344 methylation changes, in the opposite direction. The enriched terms in breast cancer included several signalling, metabolism, and developmental pathways. The Wnt signaling pathway,

346 which was altered more frequently in older breast cancer patients (Fig. 5b), showed a decrease

347 in gene expression and increase in methylation with age. In low-grade glioma, interestingly,

348 mitochondrial terms were enriched in the gene expression of younger patients. Mitochondrial 349 dysfunction is known to be important in glioma pathophysiology ${ }^{37}$, thus the different levels of 
mitochondrial aberrations might contribute to the disparities in the aggressiveness of gliomas

351 in patients of different age. We also identified numerous immune-related terms enriched across

352 several cancer types, including oesophageal, papillary renal cell, liver, and prostate cancers

353 (Supplementary Fig. 11, Supplementary Table 16). Previous studies suggested alterations in

354 immune-related gene expression and immune cell abundance changes with age in cancers ${ }^{38,39}$.

355 In the present study, we have systematically characterised the transcriptome and methylation

356 in relation to age across cancer types. Our results suggest that gene expression changes with

357 age in cancer are controlled, at least in part, by DNA methylation. These changes reflect

358 differences in biological pathways that might be important in tumour development.

\section{Discussion}

Although age is an important risk factor for cancer, how age impacts the molecular of the age-associated molecular landscape in cancer, including genomic instability, $\mathrm{LOH}$,

364 WGD, SCNAs, somatic mutations, pathway alterations, gene expression, and DNA methylation. We confirmed the known increase in mutation load ${ }^{4,5}$ and found an increase in were evident in low-grade glioma and endometrial cancer.

Cancer develops through the accumulation of genetic and epigenetic alterations.

Mutation accumulation with age is thought to be a cause of cancer and a substantial portion of

372 demonstrated in both cancer ${ }^{4,5}$ and normal tissues ${ }^{40-42}$, providing a better understanding of an

373 early carcinogenesis event. Our results show that, in addition to mutations, SCNAs, LOH and

374 WGD increase with age in several cancers, in particular low-grade glioma, endometrial and 
ovarian cancers. Recent evidence suggests that SCNA burden is a prognostic factor associated with both recurrence and death ${ }^{43}$, thus, an increased SCNA level with age might relate to poor prognosis in the elderly. The negative association between age and mutation in $I D H I$ and $A T R X$ in glioma points towards the difference of patient age at diagnosis between the $I D H$-mutant and $I D H$-WT subtypes. $I D H$-mutant tumours are observed in the majority of low-grade glioma and show favourable prognosis. IDH-WT low-grade gliomas, on the other hand, more resemble glioblastomas and have poorer survival. In glioblastoma, although $I D H$-mutants are a minority of tumours, they are also associated with younger age ${ }^{44}$. The present study together with others $^{34,45}$, therefore indicates that glioma shows unique age-associated subtypes. However, more research is needed to understand how age influences the evolution of glioma subtypes. subtypes, leading to an enrichment of hypermutated tumours, while tumours from older patients tend to harbour a higher SCNA level and lower mutation load. Previous studies have classified endometrial cancer into four subtypes: POLE, MSI-H, copy-number low and copynumber high subtypes. The POLE subtype and MSI-H subtype are dominated by the POLE and defective mismatch repair mutational signatures, respectively ${ }^{33}$. Conversely, the copynumber low and copy-number high subtypes had a dominant ageing-related mutational signature $^{31}$. The POLE and MSI-H subtypes have a favourable prognosis, while the copynumber high subtype is associated with poor survival. Therefore, endometrial cancer from younger patients is associated with POLE mutations, mismatch repair defects, high mutation load and better survival outcomes. Older endometrial cancer, however, is related to extensive SCNAs and worse prognosis. Importantly, apart from low-grade glioma and endometrial 
400 landscape in cancer driver genes and oncogenic signalling pathways. These results highlight

401 the impact of age on the molecular profile of cancer.

402

Having identified these age-related differences in the molecular landscapes of various

403 cancers, the obvious question is what drives these differences. Accumulating evidence has underscored the importance of tissue environment changes with ageing in cancer initiation and progression ${ }^{7,8,39,46}$. We reason that tissue environment changes during ageing and might provide different selective advantages for tumours harbouring different molecular alterations in turn directing the tumours to different evolutionary routes. Therefore, cancer with different genomic alterations might thrive better in younger or older patients. Gene expression and epigenetic changes related to ageing have been studied and linked to cancer $8,38,47,48$. Here, we identified numerous age-associated gene expression and corresponding DNA methylation in a

411 broad range of cancers. Indeed, age-DMGs-DEGs are those with the strongest negative

412 correlation between methylation and expression when comparing with other groups, indicating

413 that differentially expressed genes with age in cancer are partly regulated by methylation.

414 Expression and methylation changes with age link to several biological processes, showing that 415 cancer from patients with different ages present different phenotypes. We also noticed that 416 cancer in female reproductive organs including breast, ovarian and endometrial cancers are 417 among those with the highest number of age-DEGs and age-DMGs. These cancers tend to have 418 a higher mass-normalised cancer incidence, which may reflect evolutionary trade-offs 419 involving selective pressures related to reproduction ${ }^{49}$. The age-associated hormonal changes 420 could also be responsible for this age-related expression differences in cancer ${ }^{50}$. The limitation 421 of this analysis is that although we have already included tumour purity in our linear model, it

422 is not possible to account for the different tumour-constituent cell proportions and thus fully 423 exclude the influence from gene expression of non-tumor cells such as infiltrating immune 
424 cells $^{39}$. Further studies are required to provide mechanistic understanding of the impact of an

425 ageing microenvironment in shaping tumour evolution.

426 During the preparation of our manuscript, a study based on a similar concept has been

427 released by $\mathrm{Li}$ et $\mathrm{al}^{51}$. In this work, Li et al. used TCGA and the recent pan-cancer analysis of

428 whole genomes (PCAWG) data to study the age-associated genomic differences in cancer.

429 Results from the two studies are consistent on several points. Firstly, both studies indicate the

430 increase in mutations and SCNA levels with age. Next, despite using slightly different

431 statistical cutoffs and models, several age-associated genomic features are identified by both

432 studies, for example, the higher frequency of IDHI and ATRX mutations in younger glioma

433 patients. Li et al. explored mutational timing and signatures, which suggested the possible

434 underlying mechanisms for age-associated genomic differences. Our study, however, has also

435 featured an age-related genomic profile in endometrial cancer. We have investigated cancer-

436 specific associations between age and LOH, WGD and oncogenic signalling. Furthermore, we

437 have analysed age-related global transcriptomic and DNA methylation changes. Our study are

438 complementary with the $\mathrm{Li}$ et al. study, both studies thus serve as a foundation for

439 understanding age-related differences and effects on the cancer molecular landscape and

440 emphasise the importance of age in cancer genomic research that is particularly valuable in the

441 clinical practice.

442

443

444

445

446

447

448 
Methods

450

\section{Data acquisition}

451 Publicly available copy-number alteration seg files (nocnv_hg19.seg), normalized mRNA

452 expression in RSEM (.rsem.genes.normalized_results TCGA files from the legacy archive, 453 aligned to hg19), and clinical data (XML files) from TCGA were downloaded using 454 TCGAbiolinks (version 2.14.1) ${ }^{52}$. The mutation annotation format (MAF) file was downloaded 455 from the TCGA MC3 project ${ }^{53}$ (https://gdc.cancer.gov/about-data/publications/mc3-2017).

456 The somatic alterations in 10 canonical oncogenic pathways across TCGA samples were 457 obtained from a previous study by Sanchez-Vega et $\mathrm{al}^{36}$. The TCGA Illumina 458 HumanMethylation450K array data (in $\beta$-values) was downloaded from Broad GDAC 459 Firehose (http://gdac.broadinstitute.org/). The allele-specific copy number, tumour ploidy, 460 tumour purity were estimated using ASCAT (version 2.4.2) s4 $^{54}$ on hy 19 SNP6 arrays with 461 penalty $=70$ as previously described ${ }^{55,56}$. We restricted our subsequent analyses to samples that

462 have these profiles available. WGD duplication was determined using fraction of genome with 463 LOH and ploidy information. Genomic instability (GI) scores have been computed as fraction 464 of genomic regions that are not in 1+1 (for non WGD tumours) or 2+2 (for WGD tumours) 465 statuses. For each data type and each cancer type, the summary of the numbers of TCGA 466 samples included in the analysis, alongside clinical variable analysed are presented in the 467 Supplementary Table 1.

\section{Statistical analysis and visualisation}

470 Simple linear regression and multiple linear regression adjusting for clinical variables were

471 performed using the $l m$ function in $\mathrm{R}$ to access the relationship between age and continuous

472 variables of interest. Simple logistic regression to investigate the association between age and 473 binary response (e.g. mutation as 1 and wild-type as 0 ) and multiple logistic regression 
474 adjusting for covariates were carried out using the $g l m$ function in R. In pan-cancer analyses,

475 gender, race and cancer type were variables included in the linear model. Clinical variables

476 used in cancer-specific analyses included gender, race, pathologic stage, neoplasm histologic

477 grade, smoking status, alcohol consumption and cancer-specific variables such as oestrogen receptor (ER) status in breast cancer. To avoid the potential detrimental effect caused by

479 missing data, we retained only variables with missing data less than $10 \%$ of samples used in the somatic copy number alteration analysis (Supplementary Table 1). To account for the difference in the proportion of cancer cells in each tumour, tumour purity (cancer cell fraction) estimated from ASCAT was included in the linear model. When necessary, to avoid the separation problem that might occur due to the sparse-data bias ${ }^{57}$, logistf function from the logistf package (version 1.23$)^{58}$ was used to perform multivariable logistic regression with

485 Firth's penalization ${ }^{59}$. Effect sizes from logistic regression analyses were reported as odds ratio per year and $95 \%$ confidence intervals. P-values from the analyses were accounted for multiple-hypothesis testing using Benjamini-Hochberg procedure ${ }^{60}$. Statistical significance was considered if adj. p-value $<0.05$, unless specifically indicated otherwise.

All statistical analyses were carried out using R (version 3.6.3) ${ }^{61}$. Plots were generated

using ggplot2 (version 3.3.2) $)^{62}$, ggrepel (version 0.8.2) ${ }^{63}$, ggpubr (version 0.4.0) ${ }^{64}$,

ComplexHeatmap (version 2.2.0) ${ }^{65}$, and VennDiagram (version 1.6.20) ${ }^{66}$.

\section{GI score analysis}

494 GI score was calculated as a genome fraction (percent-based) that does not fit the estimated tumour ploidy, 2 for normal diploid, and 4 for tumours that have undergone the WGD process.

496 Simple linear regression was performed to identify the association between age and GI score.

497 For pan-cancer analysis, multiple linear regression was used to adjust for gender, race, and cancer type. For cancer-specific analysis, multiple linear regression accounting for clinical 
499

500

501

502

503

504

505

506

507

508

509

510

511

512

513

514

515

516

517

518

519

520

521

522

523

variables was conducted on the cancer types that had a significant association between age and GI score from the simple linear regression analysis (adj. p-value $<0.05)$. The complete set of results is presented in Supplementary Table 2.

\section{Percentage genomic LOH quantification and analysis}

To quantify the percent genomic LOH for each tumour, we used allele-specific copy number profiles from ASCAT. X and Y chromosome regions were discarded from the analysis. The $\mathrm{LOH}$ segments were segments that harbour only one allele. The percent genomic LOH was defined as 100 times the total length of LOH regions / length of the genome.

Simple linear regression and multiple linear regression adjusting for gender, race, and cancer types were conducted to investigate the relationship between age and the percent genomic LOH in the pan-cancer analysis. For cancer-specific analysis, simple linear regression was performed followed by multiple linear regression accounting for clinical factors for cancers with a significant association in simple linear regression analysis (adj. p-value $<0.05$ ). The complete set of results is in Supplementary Table 3.

\section{WGD analysis}

WGD status for each tumour was obtained from fraction of genome with $\mathrm{LOH}$ and tumour ploidy. To investigate the association between age and WGD across the pan-cancer dataset, we performed simple logistic regression and multiple logistic regression correcting for gender, race, and cancer type. For cancer-specific analysis, simple logistic regression was performed to access the association between age and WGD on tumours from each cancer type. Cancer types with a significant association between age and WGD (adj. p-value $<0.05$ ) were further subjected to the multiple logistic regression accounting for the clinical variables. The complete set of results is in Supplementary Table 4. 


\section{List of known cancer driver genes}

526 We compiled a list of known cancer driver genes from (1) the list of 243 COSMIC classic 527 genes from COSMIC database version $91^{67}$ (downloaded on $1^{\text {st }}$ July 2020), (2) the list of 260

528 significantly mutated genes from Lawrence et a ${ }^{68}$, and (3) the list of 299 cancer driver genes 529 from the TCGA Pan-Cancer study ${ }^{69}$. In total, we obtained 505 cancer genes and focused on the 530 mutations and focal-level SCNAs on these genes in our study. The full list of cancer driver 531 genes is available in Supplementary Table 8.

\section{Recurrent SCNA analysis}

534 Recurrent arm-level and focal-level SCNAs of each cancer type were identified using 535 GISTIC2.022. Segmented files (nocnv_hg19.seg) from TCGA, marker file and CNV file, 536 provided by GISTIC2.0, were used as input files. The parameters were set as follows: '537 genegistic 1 -smallmem 1 -qvt 0.25 -ta 0.25 -td 0.25 -broad 1 -brlen 0.7 -conf 0.95 -armpeel 1

538 -savegene 1'. Based on these parameters, broad events were defined as the alterations happen 539 in more than $70 \%$ of an arm. The $\log 2$ ratio thresholds for copy number gains and deletions 540 were 0.25 and -0.25 , respectively. The confidence level was set as 0.95 and the q-value was 5410.25.

To investigate the association between age and arm-level SCNAs for each cancer type,

543 simple logistic regression was performed for each chromosomal arm that was identified as recurrent SCNA in a cancer type. Only cancer types with more than 100 samples were included

545 in this analysis (Table 1). Arms with a significant association (adj. p-value $<0.05)$ were further

546 adjusted for clinical variables using multiple logistic regression. The complete set of results is

547 in Supplementary Table 6. Similarly, simple and multiple logistic regression was conducted on

548 the focal-level SCNAs for each cancer type. Regions that are not overlapped with centromeres 
549 or telomeres were removed from the analysis. The complete set of results is in Supplementary

$550 \quad$ Table 7.

551 To confirm the impact of SCNAs on gene expression, we investigated the correlation

552 between GISTIC2.0 score and RNA-seq based gene expression ( $\log 2($ normalised RSEM + 1))

553 for tumours that have both types of data using Pearson correlation. The correlation was 554 considered significant if the p-value corrected for multiple-hypothesis testing using the

555 Benjamini-Hochberg procedure $<0.05$. The complete set of results is in Supplementary Table

5567.

557

\section{SCNA score quantification and analysis}

559 Previous studies have developed the SCNA score representing the SCNA level of a tumour ${ }^{12,23}$.

560 We applied the methods described by Yuan et al ${ }^{12}$ to calculate SCNA scores. Using SCNA

561 profiles from GISTIC2.0 analysis, SCNA scores for each tumour were derived at three different

562 levels (chromosome-, arm-, and focal-level). For each tumour, each focal-event $\log 2$ copy number ratio from GISTIC2.0 was classified into the following score: 2 if the $\log 2$ ratio $\geq 1,1$

564 if the $\log 2$ ratio $<1$ and $\geq 0.25,0$ if the $\log 2$ ratio $<0.25$ and $\geq-0.25,-1$ if the $\log 2$ ratio $<$ -

5650.25 and $\geq-1$, and -2 if the $\log 2$ ratio $<-1$. The $\mid$ score $\mid$ from each focal event in a tumour was

566 then summed into a focal score of a tumour. Thereafter, the rank-based normalisation

567 (rank/number of tumours in a cancer type) was applied to focal scores from all tumours within

568 the same cancer type, resulting in normalized focal-level SCNA scores. Therefore, tumours

569 with high focal-level SCNAs will have focal-level SCNA scores close to 1, while tumours with

570 low focal-level SCNAs will have scores close to 0. For the arm- and chromosome-level SCNA

571 scores, a similar procedure was applied to the broad event $\log 2$ copy number ratio from

572 GISTIC2.0. An event was considered as a chromosome-level if both arms have the same log2

573 ratio, otherwise it was considered as an arm-level. Similar to the focal-level SCNA score, each 
arm- and chromosome-event $\log 2$ copy number ratio was classified into the 2, 1, 0, -1, -2 scores

575 using the threshold described above. The |score| from all arm-events and chromosome-events

576 for a tumour were then summed into an arm score and chromosome score, respectively. For

577 each cancer type, the rank-based normalisation was applied to arm scores and chromosome

578 scores from all tumours to derive normalised arm-level SCNA scores and normalised 579 chromosome-level SCNA scores, respectively. An overall SCNA score for a tumour was 580 defined as the sum of focal-level, arm-level, and chromosome-level SCNA scores. A

581 chromosome/arm-level SCNA score for a tumour was defined as the sum of chromosome-level

582 and arm-level SCNA scores.

The association between age and overall, chromosome/arm-level, and focal-level

584 SCNA scores for each cancer type was investigated using simple linear regression. Cancer types with a significant association $($ adj. p-value $<0.05)$ were then subjected to multiple linear regression analysis adjusting for the clinical variables. The complete set of results is included

587 in Supplementary Table 5.

\section{Analysis of age-associated somatic mutation in cancer genes}

590 We obtained the mutation data from the MAF file from the recent TCGA Multi-Center

591 Mutation Calling in Multiple Cancers (MC3) project ${ }^{53}$. In the MC3 effort, variants were called

592 using seven variant callers. We filtered the variants to keep only non-silent SNVs and indels

593 located in gene bodies, retaining only "Frame_Shift_Del", "Frame_Shift_Ins", "In_Frame_Del", “In_Frame_Ins", "Missense_Mutation", "Nonsense_Mutation", "Nonstop_Mutation", “Splice_Site" and Translation_Start_Site in the compiled list of cancer driver genes. To prevent the bias that might cause by hypermutated tumours, we restricted the analysis to tumours with $<1,000$ mutations per exome. For pan- 
599

600

601

602

603

604

605

606

607

608

609

610

611

612

613

614

615

616

617

618

619

620

621

622

cancer analysis, multiple logistic regression accounting for gender, race and cancer type was performed to investigate the association between age and mutations in 20 cancer genes that are mutated in $>5 \%$ of samples (Supplementary Table 10). For cancer-specific analysis, simple logistic regression was used to identify cancer genes that the mutations in these genes are associated with the patient's age. Only genes that are mutated in $>5 \%$ of samples from each cancer type were included in the analysis. The significant associations (adj. p-value $<0.05$ ) were further investigated using multiple logistic regression accounting for clinical variables. The complete set of results is in Supplementary Table 10.

\section{Analysis of mutational burden, MSI-H status, and POLE/POLD1 mutations}

A mutational burden was defined as the total non-silent mutations in an exome. The mutational burden for each tumour was log-transformed before using it in the subsequent analysis. To investigate the relationship between age and mutational burden in pan-cancer, multiple linear regression adjusting for gender, race and cancer type was conducted. For cancer-specific analysis, simple linear regression was performed. Cancer types with a significant association between age and mutational burden in simple linear regression analysis (adj. p-value $<0.05$ ) were further examined using multiple linear regression accounting for clinical factors. The complete set of results is in Supplementary Table 9.

Microsatellite instability status for COAD, READ, STAD, and UCEC were downloaded from TCGA using TCGAbiolinks. To study the association between the presence of high microsatellite instability (MSI-H) and age, tumours were divided into binary groups: MSI-H = TRUE and MSI-H = FALSE. Multiple logistic regression adjusting for clinical variables was then performed. Similarly, $P O L E$ and $P O L D 1$ mutation status were in a binary outcome (mutated and not mutated). Multiple logistic regression was used to investigate the 
association between age and POLE/POLD1 mutations in cancer types that contained

624 POLE/POLD1 mutations in $>5 \%$ of samples.

625

\section{Oncogenic signalling pathway analysis}

627 We used the list of pathway-level alterations in ten oncogenic pathways (cell cycle, Hippo, 628 Myc, Notch, Nrf2, PI-3-Kinase/Akt, RTK-RAS, TGF $\beta$ signaling, p53 and $\beta$-catenin/Wnt) for 629 TCGA tumours comprehensively complied by Sanchez-Vega et al ${ }^{36}$. Member genes in the 630 pathways were accessed for SCNAs, mutations, epigenetic silencing through promoter DNA

631 hypermethylation and gene fusions. We retained only the pathway alteration data of samples 632 that were presented in our SCNA analysis. For the pan-cancer analysis, we employed multiple

633 logistic regression adjusting for the patient's gender, race and cancer type to demonstrate the 634 relationship between pathway-level alteration and age. To investigate the association between 635 age and cancer-specific pathway alterations, we performed simple logistic regression. Cancer 636 types with a significant association (adj. p-value $<0.05)$ were further examined by multiple 637 logistic regression accounting for clinical variables. The complete set of results is in 638 Supplementary Table 11.

640 Gene expression and DNA methylation analysis

641 To render the results from gene expression and DNA methylation comparable, we limited the 642 analysis to genes that are presented in both types of data. The lowly expressed genes were 643 filtered out from the analysis by keeping only genes with RSEM $>0$ in more than 50 percent 644 of samples. Only protein coding genes identified using biomaRt ${ }^{70}$ (Ensembl version 100, April 645 2020). Normalised mRNA expression in RSEM for each TCGA cancer type was log2646 transformed before subjected to the multiple linear regression analysis adjusting for clinical 647 factors. RNA-seq data for colon cancer and endometrial cancer consisted of two platforms, 
648 Illumina HiSeq and Illumina GA. Thus, a platform was included as another covariate in the

649 linear regression model for these two cancer types. Genes with adj. p-value $<0.05$ were

650 considered significantly differentially expressed genes with age (age-DEGs) (Supplementary

651 Table 12). DNA methylation data was presented as $\beta$-values, which are the ratio of the

652 intensities of methylated and unmethylated alleles. Because multiple methylation probes can

653 be mapped to the same gene, we used the one-to-one mapping genes and probes by selecting

654 the probes that are most negatively correlated with the corresponding gene expression from the

655 files meth.by_min_expr_corr.data.txt. Similar multiple linear regression to the gene expression

656 analysis was performed on the methylation data. Genes with adj. p-value $<0.05$ were considered significant differentially methylated genes with age (age-DMGs). The complete set of results is in Supplementary Table 13.

The correlation between gene expression and DNA methylation was calculated using Pearson correlation. We used the Kruskal-Wallis test to investigate the differences between correlation coefficients among groups (age-DMGs-DEGs, age-DMGs, age-DEGs, other genes). The pairwise comparisons were carried out by Dunn's test. The complete set of results is in Supplementary Table 15.

Ontology (GO) terms that are enriched in tumours from younger or older patients. The analysis was done using the package ClusterProfiler (version 3.14.3) ${ }^{71}$. The complete list of enriched

GO terms is presented in Supplementary Table 16.

668

\section{Data availability}

670 TCGA data used in this study are publicly available and can be obtained from NCI's Genomic

671 Data Commons portal (https://portal.gdc.cancer.gov/), TCGAbiolinks (version 2.14.1) ${ }^{52}$ and

672 Broad GDAC Firehose (http://gdac.broadinstitute.org/). 


\section{Code availability}

675 The custom scripts for data analysis and generate figures are available at

676 https://github.com/maglab/Age-associated_cancer_genome.

677

678

679

680

681

682

683

684

685

686

687

688

689

690

691

692

693

694

695

696

697 


\section{References}

6991 de Magalhaes, J. P. How ageing processes influence cancer. Nat Rev Cancer 13, 357365, doi:10.1038/nrc3497 (2013).

7012 Laconi, E., Marongiu, F. \& DeGregori, J. Cancer as a disease of old age: changing mutational and microenvironmental landscapes. $B r J$ Cancer 122, 943-952, doi:10.1038/s41416-019-0721-1 (2020).

3 Nowell, P. C. The clonal evolution of tumor cell populations. Science 194, 23-28, doi:10.1126/science.959840 (1976).

7064 Milholland, B., Auton, A., Suh, Y. \& Vijg, J. Age-related somatic mutations in the cancer genome. Oncotarget 6, 24627-24635, doi:10.18632/oncotarget.5685 (2015).

7085 Alexandrov, L. B. et al. Clock-like mutational processes in human somatic cells. Nat Genet 47, 1402-1407, doi:10.1038/ng.3441 (2015).

6 Tomasetti, C., Vogelstein, B. \& Parmigiani, G. Half or more of the somatic mutations in cancers of self-renewing tissues originate prior to tumor initiation. Proc Natl Acad Sci U S A 110, 1999-2004, doi:10.1073/pnas.1221068110 (2013).

7 Fane, M. \& Weeraratna, A. T. How the ageing microenvironment influences tumour progression. Nat Rev Cancer 20, 89-106, doi:10.1038/s41568-019-0222-9 (2020).

8 Chatsirisupachai, K., Palmer, D., Ferreira, S. \& de Magalhaes, J. P. A human tissuespecific transcriptomic analysis reveals a complex relationship between aging, cancer, and cellular senescence. Aging Cell 18, e13041, doi:10.1111/acel.13041 (2019).

9 Li, C. H., Haider, S., Shiah, Y. J., Thai, K. \& Boutros, P. C. Sex Differences in Cancer Driver Genes and Biomarkers. Cancer Res 78, 5527-5537, doi:10.1158/00085472.CAN-18-0362 (2018).

10 Yuan, Y. et al. Comprehensive Characterization of Molecular Differences in Cancer between Male and Female Patients. Cancer Cell 29, 711-722, doi:10.1016/j.ccell.2016.04.001 (2016).

11 Sinha, S. et al. Higher prevalence of homologous recombination deficiency in tumors from African Americans versus European Americans. Nature Cancer 1, 112-121, doi:10.1038/s43018-019-0009-7 (2020).

12 Yuan, J. et al. Integrated Analysis of Genetic Ancestry and Genomic Alterations across Cancers. Cancer Cell 34, 549-560 e549, doi:10.1016/j.ccell.2018.08.019 (2018).

13 Ma, X. et al. Pan-cancer genome and transcriptome analyses of 1,699 paediatric leukaemias and solid tumours. Nature 555, 371-376, doi:10.1038/nature25795 (2018).

14 Grobner, S. N. et al. The landscape of genomic alterations across childhood cancers. Nature 555, 321-327, doi:10.1038/nature25480 (2018).

15 Brennan, C. W. et al. The somatic genomic landscape of glioblastoma. Cell 155, 462477, doi:10.1016/j.cell.2013.09.034 (2013).

16 Gerhauser, C. et al. Molecular Evolution of Early-Onset Prostate Cancer Identifies Molecular Risk Markers and Clinical Trajectories. Cancer Cell 34, 996-1011 e1018, doi:10.1016/j.ccell.2018.10.016 (2018).

17 Liao, S. et al. The molecular landscape of premenopausal breast cancer. Breast Cancer Res 17, 104, doi:10.1186/s13058-015-0618-8 (2015).

18 Ryland, G. L. et al. Loss of heterozygosity: what is it good for? BMC Med Genomics 8, 45, doi:10.1186/s12920-015-0123-z (2015).

19 Lopez, S. et al. Interplay between whole-genome doubling and the accumulation of deleterious alterations in cancer evolution. Nat Genet 52, 283-293, doi:10.1038/s41588-020-0584-7 (2020).

20 Bielski, C. M. et al. Genome doubling shapes the evolution and prognosis of advanced cancers. Nat Genet 50, 1189-1195, doi:10.1038/s41588-018-0165-1 (2018). 
21 Van de Peer, Y., Mizrachi, E. \& Marchal, K. The evolutionary significance of polyploidy. Nat Rev Genet 18, 411-424, doi:10.1038/nrg.2017.26 (2017).

22 Mermel, C. H. et al. GISTIC2.0 facilitates sensitive and confident localization of the targets of focal somatic copy-number alteration in human cancers. Genome Biol 12, R41, doi:10.1186/gb-2011-12-4-r41 (2011).

23 Davoli, T., Uno, H., Wooten, E. C. \& Elledge, S. J. Tumor aneuploidy correlates with markers of immune evasion and with reduced response to immunotherapy. Science $\mathbf{3 5 5}$, doi:10.1126/science.aaf8399 (2017).

24 Korber, V. et al. Evolutionary Trajectories of IDH(WT) Glioblastomas Reveal a Common Path of Early Tumorigenesis Instigated Years ahead of Initial Diagnosis. Cancer Cell 35, 692-704 e612, doi:10.1016/j.ccell.2019.02.007 (2019).

$25 \mathrm{Xu}, \mathrm{F}$. et al. Elevated expression of RIT1 correlates with poor prognosis in endometrial cancer. Int J Clin Exp Pathol 8, 10315-10324 (2015). Bonneville, R. et al. Landscape of Microsatellite Instability Across 39 Cancer Types. JCO Precis Oncol 2017, doi:10.1200/PO.17.00073 (2017).

Kim, T. M., Laird, P. W. \& Park, P. J. The landscape of microsatellite instability in colorectal and endometrial cancer genomes. Cell 155, 858-868, doi:10.1016/j.cell.2013.10.015 (2013).

28 Chalmers, Z. R. et al. Analysis of 100,000 human cancer genomes reveals the landscape of tumor mutational burden. Genome Med 9, 34, doi:10.1186/s13073-017-0424-2 (2017).

29 Campbell, B. B. et al. Comprehensive Analysis of Hypermutation in Human Cancer. Cell 171, 1042-1056 e1010, doi:10.1016/j.cell.2017.09.048 (2017).

30 Shlien, A. et al. Combined hereditary and somatic mutations of replication error repair genes result in rapid onset of ultra-hypermutated cancers. Nat Genet 47, 257-262, doi:10.1038/ng.3202 (2015).

31 Ashley, C. W. et al. Analysis of mutational signatures in primary and metastatic endometrial cancer reveals distinct patterns of DNA repair defects and shifts during tumor progression. Gynecol Oncol 152, 11-19, doi:10.1016/j.ygyno.2018.10.032 (2019).

32 Berger, A. C. et al. A Comprehensive Pan-Cancer Molecular Study of Gynecologic and Breast Cancers. Cancer Cell 33, 690-705 e699, doi:10.1016/j.ccell.2018.03.014 (2018).

33 Cancer Genome Atlas Research, N. et al. Integrated genomic characterization of endometrial carcinoma. Nature 497, 67-73, doi:10.1038/nature12113 (2013).

34 Yan, H. et al. IDH1 and IDH2 mutations in gliomas. $N$ Engl $J$ Med 360, 765-773, doi:10.1056/NEJMoa0808710 (2009).

35 Cancer Genome Atlas Research, N. et al. Comprehensive, Integrative Genomic Analysis of Diffuse Lower-Grade Gliomas. $N$ Engl $J$ Med 372, 2481-2498, doi:10.1056/NEJMoa1402121 (2015).

36 Sanchez-Vega, F. et al. Oncogenic Signaling Pathways in The Cancer Genome Atlas. Cell 173, 321-337 e310, doi:10.1016/j.cell.2018.03.035 (2018).

37 Ordys, B. B., Launay, S., Deighton, R. F., McCulloch, J. \& Whittle, I. R. The role of mitochondria in glioma pathophysiology. Mol Neurobiol 42, 64-75, doi:10.1007/s12035-010-8133-5 (2010).

$38 \mathrm{Wu}, \mathrm{Y}$. et al. Comprehensive transcriptome profiling in elderly cancer patients reveals aging-altered immune cells and immune checkpoints. Int J Cancer 144, 1657-1663, doi:10.1002/ijc.31875 (2019).

39 Erbe, R. et al. Aging interacts with tumor biology to produce major changes in the immune tumor microenvironment. bioRxiv, doi:https://doi.org/10.1101/2020.06.08.140764 (2020). 
40 Martincorena, I. et al. Somatic mutant clones colonize the human esophagus with age. Science 362, 911-917, doi:10.1126/science.aau3879 (2018).

41 Martincorena, I. et al. Tumor evolution. High burden and pervasive positive selection of somatic mutations in normal human skin. Science 348, 880-886, doi:10.1126/science.aaa6806 (2015).

42 Xie, M. et al. Age-related mutations associated with clonal hematopoietic expansion and malignancies. Nat Med 20, 1472-1478, doi:10.1038/nm.3733 (2014).

43 Hieronymus, H. et al. Tumor copy number alteration burden is a pan-cancer prognostic factor associated with recurrence and death. Elife 7, doi:10.7554/eLife.37294 (2018).

44 Mirchia, K. \& Richardson, T. E. Beyond IDH-Mutation: Emerging Molecular Diagnostic and Prognostic Features in Adult Diffuse Gliomas. Cancers (Basel) 12, doi:10.3390/cancers12071817 (2020).

45 Verhaak, R. G. et al. Integrated genomic analysis identifies clinically relevant subtypes of glioblastoma characterized by abnormalities in PDGFRA, IDH1, EGFR, and NF1. Cancer Cell 17, 98-110, doi:10.1016/j.ccr.2009.12.020 (2010).

46 Rozhok, A. \& DeGregori, J. A generalized theory of age-dependent carcinogenesis. Elife 8, doi:10.7554/eLife.39950 (2019).

47 Perez, R. F., Tejedor, J. R., Bayon, G. F., Fernandez, A. F. \& Fraga, M. F. Distinct chromatin signatures of DNA hypomethylation in aging and cancer. Aging Cell 17, e12744, doi:10.1111/acel.12744 (2018).

48 Johnson, A. A. et al. The role of DNA methylation in aging, rejuvenation, and agerelated disease. Rejuvenation Res 15, 483-494, doi:10.1089/rej.2012.1324 (2012).

49 Silva, A. S. et al. Gathering insights on disease etiology from gene expression profiles of healthy tissues. Bioinformatics 27, 3300-3305, doi:10.1093/bioinformatics/btr559 (2011).

50 Benz, C. C. Impact of aging on the biology of breast cancer. Crit Rev Oncol Hematol 66, 65-74, doi:10.1016/j.critrevonc.2007.09.001 (2008).

51 Li, C. H., Haider, S. \& Boutros, P. C. Age Influences on the Molecular Presentation of Tumours. bioRxiv, doi:https://doi.org/10.1101/2020.07.07.192237 (2020).

52 Colaprico, A. et al. TCGAbiolinks: an R/Bioconductor package for integrative analysis of TCGA data. Nucleic Acids Res 44, e71, doi:10.1093/nar/gkv1507 (2016).

53 Ellrott, K. et al. Scalable Open Science Approach for Mutation Calling of Tumor Exomes Using Multiple Genomic Pipelines. Cell Syst 6, 271-281 e277, doi:10.1016/j.cels.2018.03.002 (2018).

54 Van Loo, P. et al. Allele-specific copy number analysis of tumors. Proc Natl Acad Sci U S A 107, 16910-16915, doi:10.1073/pnas.1009843107 (2010).

55 Martincorena, I. et al. Universal Patterns of Selection in Cancer and Somatic Tissues. Cell 171, 1029-1041 e1021, doi:10.1016/j.cell.2017.09.042 (2017).

56 Alexandrov, L. B. et al. Mutational signatures associated with tobacco smoking in human cancer. Science 354, 618-622, doi:10.1126/science.aag0299 (2016).

57 Greenland, S., Mansournia, M. A. \& Altman, D. G. Sparse data bias: a problem hiding in plain sight. BMJ 352, i1981, doi:10.1136/bmj.i1981 (2016).

58 Heinze, G. \& Ploner, M. logistf: Firth's Bias-Reduced Logistic Regression. (2018).

59 Heinze, G. \& Schemper, M. A solution to the problem of separation in logistic regression. Stat Med 21, 2409-2419, doi:10.1002/sim.1047 (2002).

60 Benjamini, Y. \& Hochberg, Y. Controlling the False Discovery Rate: A Practical and Powerful Approach to Multiple Testing. J. R. Statist. Soc. B 57, 289-300, doi:10.1111/j.2517-6161.1995.tb02031.x (1995).

61 Team, R. C. R: A Language and Environment for Statistical Computing. (2020). 
84662 Wickham, H. ggplot2: Elegant Graphics for Data Analysis. (Springer-Verlag New York, 2016).

84863 Slowikowski, K. ggrepel: Automatically Position Non-Overlapping Text Labels with

849 'ggplot2'. (2020).

. ggpubr: 'gg multidimensional genomic data. doi:10.1093/bioinformatics/btw313 (2016).

66 Chen, H. \& Boutros, P. C. VennDiagram: a package for the generation of highlycustomizable Venn and Euler diagrams in R. BMC Bioinformatics 12, 35, doi:10.1186/1471-2105-12-35 (2011).

856

857

858

859

860

861

67 Tate, J. G. et al. COSMIC: the Catalogue Of Somatic Mutations In Cancer. Nucleic Acids Res 47, D941-D947, doi:10.1093/nar/gky1015 (2019).

68 Lawrence, M. S. et al. Discovery and saturation analysis of cancer genes across 21 tumour types. Nature 505, 495-501, doi:10.1038/nature12912 (2014).

69 Bailey, M. H. et al. Comprehensive Characterization of Cancer Driver Genes and Mutations. Cell 173, 371-385 e318, doi:10.1016/j.cell.2018.02.060 (2018).

70 Durinck, S., Spellman, P. T., Birney, E. \& Huber, W. Mapping identifiers for the integration of genomic datasets with the R/Bioconductor package biomaRt. Nat Protoc 4, 1184-1191, doi:10.1038/nprot.2009.97 (2009).

71 Yu, G., Wang, L. G., Han, Y. \& He, Q. Y. clusterProfiler: an R package for comparing biological themes among gene clusters. OMICS 16, 284-287, doi:10.1089/omi.2011.0118 (2012).

\section{Acknowledgements}

871 K.C. is supported by a Mahidol-Liverpool PhD scholarship from Mahidol University,

872 Thailand, and the University of Liverpool, UK. J.P.M. is grateful to funding from the Wellcome

873 Trust (208375/Z/17/Z) and the Biotechnology and Biological Sciences Research Council

874 (BB/R014949/1). This work was supported by the Francis Crick Institute, which receives its

875 core funding from Cancer Research UK (FC001202), the UK Medical Research Council

876 (FC001202), and the Wellcome Trust (FC001202). P.V.L. is a Winton Group Leader in

877 recognition of the Winton Charitable Foundation's support towards the establishment of The

878 Francis Crick Institute. We wish to thank members of the Integrative Genomics of Ageing

879 Group for suggestions and discussion. 
884 K.C., T.L., P.V.L. and J.P.M. conceived the project and designed the study. T.L. and P.V.L.

885 provided data. K.C. performed the analyses with helps from T.L. T.L., L.P., P.V.L. and J.P.M.

886 provided critical insights and were involved in data interpretation. K.C. wrote the first draft of

887 the manuscript. All authors edited and approved the manuscript.

\section{8}

889

890

891

892

893

894

895

896

897

898

899

900

901

902

903

904

905

906

907 
bioRxiv preprint doi: https://doi.org/10.1101/2020.08.25.266403; this version posted August 25, 2020. The copyright holder for this preprint (which was not certified by peer review) is the author/funder, who has granted bioRxiv a license to display the preprint in perpetuity. It is made available under aCC-BY 4.0 International license.

908 Table 1 Summary of TCGA cancer type and number of samples used in each analysis

\begin{tabular}{|c|c|c|c|c|c|c|c|}
\hline Cancer type & Abbreviation & $\begin{array}{l}\text { GI, LOH, } \\
\text { WGD }\end{array}$ & SCNAs & $\begin{array}{l}\text { Mutations } \\
\text { (hypermutat } \\
\text { ed tumours } \\
\text { removed) }\end{array}$ & $\begin{array}{l}\text { Pathway } \\
\text { alterations }\end{array}$ & $\begin{array}{c}\text { Gene } \\
\text { expression }\end{array}$ & $\begin{array}{c}\text { DNA } \\
\text { methylation }\end{array}$ \\
\hline Adrenocortical carcinoma & $\mathrm{ACC}$ & 89 & 89 & $89(88)$ & 76 & 77 & 78 \\
\hline Bladder Urothelial Carcinoma & BLCA & 370 & 369 & $369(364)$ & 361 & 366 & 370 \\
\hline Breast invasive carcinoma & BRCA & 1015 & 1011 & $954(946)$ & 922 & 1011 & 719 \\
\hline $\begin{array}{c}\text { Cervical squamous cell } \\
\text { carcinoma and endocervical } \\
\text { adenocarcinoma }\end{array}$ & CESC & 287 & 287 & $271(263)$ & 264 & 284 & 287 \\
\hline Cholangiocarcinoma & $\mathrm{CHOL}$ & 35 & 35 & $35(35)$ & 35 & 35 & 35 \\
\hline Colon adenocarcinoma & COAD & 411 & 411 & $374(319)$ & 323 & 410 & 278 \\
\hline $\begin{array}{l}\text { Lymphoid Neoplasm Diffuse } \\
\text { Large B-cell Lymphoma }\end{array}$ & DLBC & 42 & 42 & $32(32)$ & 32 & 42 & 42 \\
\hline Oesophageal carcinoma & ESCA & 176 & 176 & $176(174)$ & 165 & 175 & 176 \\
\hline Glioblastoma multiforme & GBM & 489 & 489 & $356(354)$ & 116 & 137 & 259 \\
\hline $\begin{array}{c}\text { Head and Neck squamous cell } \\
\text { carcinoma }\end{array}$ & HNSC & 489 & 489 & $472(469)$ & 459 & 481 & 489 \\
\hline Kidney Chromophobe & $\mathrm{KICH}$ & 66 & 66 & $66(66)$ & 65 & 66 & 66 \\
\hline $\begin{array}{c}\text { Kidney renal clear cell } \\
\text { carcinoma }\end{array}$ & KIRC & 496 & 496 & $343(343)$ & 331 & 493 & 296 \\
\hline $\begin{array}{c}\text { Kidney renal papillary cell } \\
\text { carcinoma }\end{array}$ & KIRP & 228 & 228 & $222(222)$ & 215 & 228 & 213 \\
\hline Acute Myeloid Leukaemia & LAML & 126 & 121 & $55(54)$ & 101 & 102 & 121 \\
\hline Brain Lower Grade Glioma & LGG & 488 & 488 & $484(484)$ & 482 & 488 & 488 \\
\hline Liver hepatocellular carcinoma & LIHC & 355 & 355 & $342(340)$ & 334 & 349 & 355 \\
\hline Lung adenocarcinoma & LUAD & 460 & 460 & $456(438)$ & 446 & 456 & 402 \\
\hline Lung squamous cell carcinoma & LUSC & 460 & 460 & $444(437)$ & 426 & 457 & 336 \\
\hline Mesothelioma & MESO & 82 & 82 & $77(77)$ & 77 & 82 & 82 \\
\hline $\begin{array}{c}\text { Ovarian serous } \\
\text { cystadenocarcinoma }\end{array}$ & $\mathrm{OV}$ & 556 & 556 & $397(395)$ & 173 & 288 & 545 \\
\hline Pancreatic adenocarcinoma & PAAD & 133 & 133 & $130(129)$ & 113 & 127 & 132 \\
\hline $\begin{array}{c}\text { Pheochromocytoma and } \\
\text { Paraganglioma }\end{array}$ & PCPG & 165 & 157 & $165(164)$ & 154 & 165 & 165 \\
\hline Prostate adenocarcinoma & PRAD & 434 & 434 & $434(432)$ & 425 & 434 & 434 \\
\hline Rectum adenocarcinoma & READ & 152 & 152 & $132(127)$ & 109 & 151 & 95 \\
\hline Sarcoma & SARC & 229 & 229 & $213(211)$ & 209 & 227 & 229 \\
\hline Skin Cutaneous Melanoma & SKCM & 434 & 434 & $432(340)$ & 332 & 433 & 434 \\
\hline Stomach adenocarcinoma & STAD & 388 & 388 & $385(345)$ & 340 & 365 & 341 \\
\hline Testicular Germ Cell Tumours & TGCT & 129 & 129 & $124(124)$ & 123 & 129 & 129 \\
\hline Thyroid carcinoma & THCA & 260 & 260 & $249(248)$ & 244 & 259 & 258 \\
\hline Thymoma & THYM & 76 & 76 & $76(76)$ & 73 & 73 & 76 \\
\hline $\begin{array}{c}\text { Uterine Corpus Endometrial } \\
\text { Carcinoma }\end{array}$ & UCEC & 434 & 434 & $421(366)$ & 406 & 432 & 360 \\
\hline Uterine Carcinosarcoma & UCS & 52 & 52 & $52(51)$ & 52 & 52 & 52 \\
\hline Uveal Melanoma & UVM & 72 & 72 & $72(72)$ & 72 & 72 & 72 \\
\hline \multicolumn{2}{|l|}{ Total } & 9678 & 9660 & $8899(8585)$ & 8055 & 8946 & 8414 \\
\hline
\end{tabular}




\section{$910 \quad$ Figure legends}

911 Fig. 1 Association between cancer patients' age and genomic instability (GI) score, percent

912 genomic loss-of-heterozygosity (LOH) and whole-genome duplication events (WGD). a

913 Association between age and pan-cancer GI score. Dots are coloured by cancer type. Multiple

914 linear regression R-squared and p-value are shown in the figure. b Association between age

915 and cancer type-specific GI score. Linear regression coefficients and significant values are

916 shown in the figure. Cancers with a significant positive association between age and GI score

917 after using multiple linear regression (adj. p-value $<0.05$ ) are highlighted in red. Cancers with

918 a significant association in simple linear regression but not significant after using multiple

919 linear regression are showed in black. The grey line indicates adj. p-value $=0.05$. Dot size is

920 proportional to median GI score. c Association between age and pan-cancer percent genomic

921 LOH. Dots are coloured by cancer type. Multiple linear regression R-squared and p-value are

922 shown in the figure. d Association between age and cancer type-specific percent genomic

923 LOH. Simple linear regression results are shown. Cancers with a significant positive and

924 negative association between age and percent genomic LOH after using multiple linear regression are highlighted in red and blue, respectively. Cancer with a significant association in simple linear regression but not significant after using multiple linear regression is showed

927 in black. The grey line indicates adj. $p$-value $=0.05$. Dot size is proportional to median percent

928 genomic LOH. e Association between age and WGD events in pan-cancer, OV, and UCEC.

929 Multiple logistic regression p-values were indicated in the figure.

931 Fig. 2 Association between cancer patients' age and somatic copy-number alterations

932 (SCNAs). Volcano plot representing the association between age and (a) overall, (b) focal-

933 level and (c) chromosome/arm-level SCNA scores. Linear regression coefficients and

934 significant values are shown. Cancers with a significant positive and negative association 
between age and SCNA score after using multiple linear regression (adj. p-value $<0.05$ ) are regression but not significant after using multiple linear regression are showed in black. The

938 grey line indicates adj. $\mathrm{p}$-value $=0.05$. Dot size is proportional to median SCNA score. $\mathbf{d}, \mathbf{e}$

939 The left and right dot plots show the association between age and arm-level copy-number gains

940 and copy-number deletions. Circle size corresponds to the significant level, red and blue

941 represent positive and negative associations, respectively. f, g Heatmaps represent recurrently

942 gain and deletion arms in LGG and UCEC, respectively. Samples are sorted by age. Colours

943 represent copy-number changes from GISTIC2.0, blue denotes deletion and red corresponds

944 to gain.

945

946 Fig. 3 Association between cancer patients' age and focal-level SCNAs. a Number of gained

947 and deleted focal regions that showed a significant association with age per cancer type

948 (multiple logistic regression, adj. p-value <0.05). Heatmap showing age-associated focal-level

949 SCNAs in (b) LGG and (c) UCEC. Samples are sorted by age. Colours represent copy-number 950 changes from GISTIC2.0, blue denotes deletion and red corresponds to gain. The gain_or_loss

951 legend demonstrates that the region is recurrently gained or deleted. The direction legend

952 shows whether the gain/deletion of the region increases or decreases with age. d Age-

953 associated SCNA changes in cancer driver genes. Cancer driver genes located in the age-

954 associated focal regions are plotted by cancer type. Colours of the dot represent the condition

955 of the focal region where the gene located in as follows: blue - decrease deletion; green -

956 increase deletion; yellow - decrease gain; and red - increase gain with age. e The effect of copy-

957 number changes on gene expression of $C D K N 2 A$ in $\mathrm{LGG}, M Y C$ in $\mathrm{OV}, C R E B B P$ and $R I T 1$ in

958 UCEC. These are examples of genes with age-associated changes in SCNAs. Violin plots show 
959 the $\log 2$ (normalized expression +1 ) of samples grouped by their SCNA status. Pearson

960 correlation coefficient $r$ and $p$-value are shown in the figures.

961

962 Fig. 4 Association between cancer patients' age and somatic mutations. a The proportion of

963 hypermutated tumours $(>1,000$ mutations/exome) in young (age $\leq 50)$ and old (age $>50)$

964 UCEC. The statistical significant (p-value) was calculated using Fisher's exact test. b The association between age and MSI-H in UCEC. The statistical significance was calculated from

966 the multiple logistic regression adjusting for clinical variables. The p-value is shown in the

967 figure. $\mathbf{c}$ The association between age and POLE/POLD1 mutations in UCEC. The statistical

968 significance (p-value) was calculated from the multiple logistic regression adjusting for clinical

969 variables. d A pan-cancer association between age and mutations. Multiple logistic regression

970 coefficient and significant values are shown. Genes with a significant positive and negative

971 association between age and somatic mutations after using multiple logistic regression (adj. p-

972 value $<0.05)$ are highlighted in red and blue, respectively. e Summary of the cancer type-

973 specific association between age and mutations. Multiple logistic regression coefficient and

974 significant values are shown. Only genes with a significant association (adj. p-value $<0.05)$

975 are shown in the figure. A colour code is provided to denote the cancer type where the

976 association between age and gene mutation was found. f Heatmap showing age-associated

977 mutations in GBM and LGG. Samples are sorted by age. Colours represent types of mutation.

978 The right annotation legend indicates the direction of change, increase or decrease mutations

979 with age. The mutational burden of samples is presented in the dot above the heatmap.

980

981 Fig. 5 Association between cancer patients' age and oncogenic signalling pathway alterations.

982 a Association between age and oncogenic pathway alterations in the pan-cancer level. Multiple

983 logistic regression coefficients and significant values are shown. Pathways with a significant 
positive association between age and alterations (adj. p-value $<0.05)$ are highlighted in red. $\mathbf{b}$

985 Cancer-specific age-associated pathway alterations. Pathways that show a significant positive and negative association with age per cancer type (multiple logistic regression, adj. p-value $<$

987 0.05) are displayed in red and blue dots, respectively. c Heatmap showing age-associated alterations in genes associated with TP53 and cell cycle pathways in LGG. Samples are sorted by age. Colours represent types of alteration.

990

Fig. 6 Age-related gene expression in cancers was controlled by age-related methylation. a

992 Number of age-DEGs and age-DMGs across cancer types. Red dots represent up-regulated

993 genes, while blue dots denote down-regulated genes. The dot size corresponds to the number of genes. b Venn diagrams of the overlap between age-DEGs and age-DMGs. LGG and BRCA are shown as examples. Venn diagrams of the other cancers are shown in Supplementary Fig.

9. c The distribution of overlap genes between age-DMGs and age-DEGs. The genes were classified into (1) down-regulated methylation and down-regulated expression, (2) downregulated methylation and up-regulated expression, (3) up-regulated methylation and downregulated expression, and (4) up-regulated methylation and up-regulated expression. $\mathbf{d}$ Violin plots showing the distribution of the Pearson correlation coefficient between methylation and gene expression in LGG and BRCA. Genes were grouped into (1) common genes between ageDMGs and age-DEGs (age-DMGs-DEGs), (2) age-DMGs only genes, (3) age-DEGs only genes, and (4) other genes. The group comparison was performed by the Kruskal-Wallis test. The pairwise comparisons were done using Dunn's test. P-values from Dunn's test between age-DMGs-DEGs and the other groups are shown. The plots for the other cancers are shown in Supplementary Fig. 10. e The enriched gene ontology (GO) terms identified by GSEA in

1007 LGG and BRCA. The dot size corresponds to a significant level. A GO term was considered 1008 significantly enriched term if adj. p-value $<0.05$ for gene expression and adj. $p$-value $<0.1$ for 
methylation. Colours represent enrichment scores, red denotes positive score (enriched in older other cancers are shown in Supplementary Fig. 11.

1012

1013 Supplementary Fig. 1 Association between age and (a) GI score and (b) percent genomic

LOH. Multiple linear regression was performed to identify the relationship between age and

1015

GI score or percent genomic LOH for each cancer type. Cancer types with a significant association (adj. p-value $<0.05$ ) are shown together with adjusted $\mathrm{R}$-squared and $\mathrm{p}$-values from multiple linear regression analysis.

1018

Supplementary Fig. 2 Association between age and (a) overall SCNA score, (b) regression was performed to identify the relationship between age and SCNA score for each cancer type. Cancer types with a significant association (adj. p-value $<0.05$ ) are shown together with adjusted R-squared and p-value from multiple linear regression analysis.

Supplementary Fig. 3 Heatmaps represent recurrent gain and deletion of arms across cancer types. Samples are sorted by age. Colours represent copy-number changes from GISTIC2.0, blue denotes deletion and red corresponds to gain.

Supplementary Fig. 4 Heatmaps represent recurrent gain and deletion of focal-regions that decreases with age. 
1035 Supplementary Fig. 5 Association between age and mutational burden. Simple linear 1036 regression was performed to investigate the association between age and mutational burden.

1037 Cancer types with a significant association (adj. p-value $<0.05$ ) were further investigated using multiple linear regression. The figure shows pan-cancer analysis and cancer type-specific analysis for every cancer that had a significant association in the simple linear regression analysis. Adjusted R-squared and p-value from multiple linear regression analysis are

1041 displayed.

Supplementary Fig. 6 Association between age and (a) MSI-H in COAD, READ, and STAD, and (b) POLE/POLD1 mutations in cancer types containing the mutations in these genes in more than $5 \%$ of the samples. The statistical significance ( $p$-value showed) was calculated from the multiple logistic regression adjusting for clinical variables.

Supplementary Fig. 7 Heatmap showing age-associated mutations in 11 cancer types. Samples are sorted by age. Colours represent types of mutation. The right annotation legend indicates the direction of change, increase or decrease mutations with age. The mutational burden of samples is presented in the dot above the heatmap.

Supplementary Fig. 8 Pearson correlation between linear regression coefficient of age on DNA methylation level and linear regression coefficient of age on gene expression. The regression coefficients were obtained from the multiple linear regression analysis to investigate the association between age and DNA methylation or gene expression. Pearson correlation coefficient and p-values are shown. 
Supplementary Fig. 9 Venn diagrams of the overlap between age-DEGs and age-DMGs in 8 methylation with age (Methy_Up) and genes with decreased methylation with age

1063 (Methy_Down).

Supplementary Fig. 10 Pearson correlation coefficient between methylation and gene expression. a Violin plots showing the distribution of the Pearson correlation coefficient between methylation and gene expression in 8 cancer types. Genes were grouped into (1) overlapping genes between age-DMGs and age-DEGs (age-DMGs-DEGs), (2) age-DMGs only genes, (3) age-DEGs only genes, and (4) other genes. The group comparison was performed by the Kruskal-Wallis test. The pairwise comparisons were done using Dunn's test. P-values from Dunn's test between age-DMGs-DEGs and the other groups are shown. b

1072 Density plots showing the distribution of the Pearson correlation coefficient between methylation and gene expression across 10 cancer types.

Supplementary Fig. 11 The enriched age-related gene ontology (GO) terms identified by considered significantly enriched if adj. p-value $<0.05$ for gene expression and adj. p-value $<$ in older patients), while blue signifies negative score (enriched in younger patients). No enriched term was identified from the gene expression data of UCEC. 
List of supplementary tables

1085

Supplementary Table 1: Summary of the number of samples and clinical variables used in

the study

1087 Supplementary Table 2: Association between age and GI scores

1088 Supplementary Table 3: Association between age and percent genomic LOH

1089 Supplementary Table 4: Association between age and WGD

1090 Supplementary Table 5: Association between age and SCNA scores

1091 Supplementary Table 6: Association between age and arm-level SCNAs

1092 Supplementary Table 7: Association between age and focal-level SCNAs

1093 Supplementary Table 8: List of previously identified cancer driver genes

1094 Supplementary Table 9: Association between age and mutational burden

1095 Supplementary Table 10: Association between age and somatic mutations

1096 Supplementary Table 11: Association between age and oncogenic signalling pathway

1097 Supplementary Table 12: Gene expression changes with age

1098 Supplementary Table 13: DNA methylation changes with age

1099 Supplementary Table 14: Number of overlapping genes between age-DEGs and age-DMGs

1100 Supplementary Table 15: Comparision of the correlation between methylation and gene

1101 expression in age-DMGs-DEGs, age-DMGs, age-DEGs and other genes

1102 Supplementary Table 16: List of enriched GO terms identified using GSEA 
bioRxiv preprint doi: https://doi.org/10.1101/2020.08 25.266403; this version posted August 25, 2020. The copyright holder for this preprint (which was not certified by peer review) is the author/funder, who has granted bioRxiv a license to display the preprint in perpetuity. It is made available under aCC-BY 4.0 International license.

a

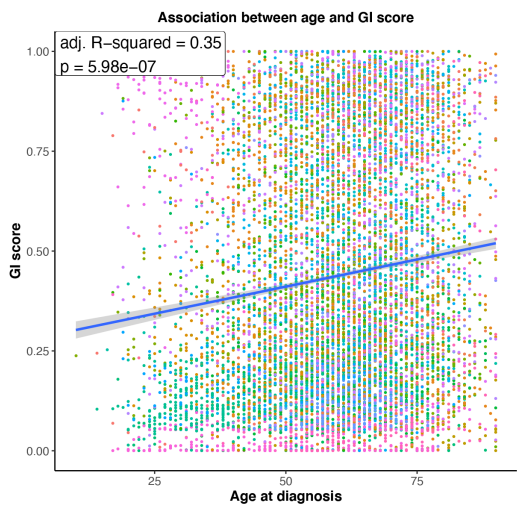

C

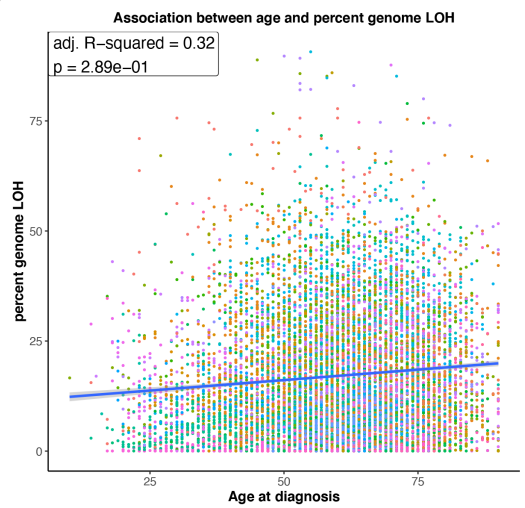

e

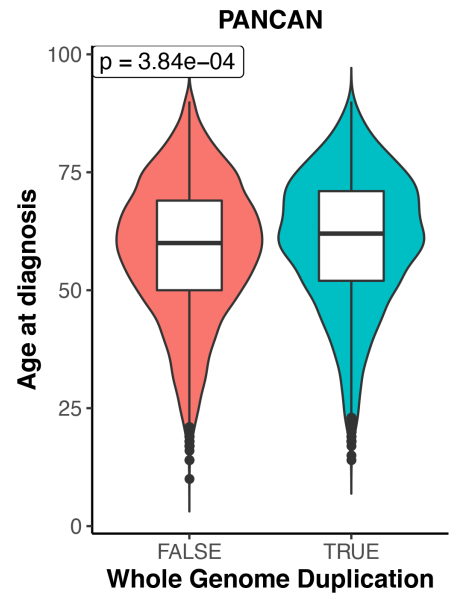

ACC. Lusc

BLCA $:$ MESO
BRCA $:$ OV

CHOL . PCPG

\begin{tabular}{lll} 
COAD & PAAD \\
DLBC & READ \\
\hline
\end{tabular}

\begin{tabular}{lll} 
EECA & SARC \\
GBM & SKCM \\
\hline &
\end{tabular}

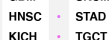

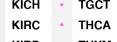

KIRP THYM
LAML UCEC

LAML UCEC
LGG UCS
LHC UVM

LHAC
LUAD

ACC LUSC

$\begin{aligned} & \text { BLCA } \\ & \text { BRCA }\end{aligned}:$ MESO

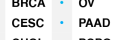

CHOL
COAD PCPG
PRAD

$\begin{array}{lll}\text { COAD } & \text { PRAD } \\ \text { DLBC } & \text { READ } \\ \text { ISSCA } & \text { SARC }\end{array}$

$\begin{array}{lll}\text { ESCA } & \text { SARC } \\ \text { GBM } & \text { SKCM }\end{array}$

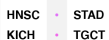

KICH TGCT
KIRC THCA

\begin{tabular}{lll} 
KIRP & THYM \\
LAML & UCEC \\
\hline
\end{tabular}

LAG UCS
LHC UVM

LHC
LUAD b

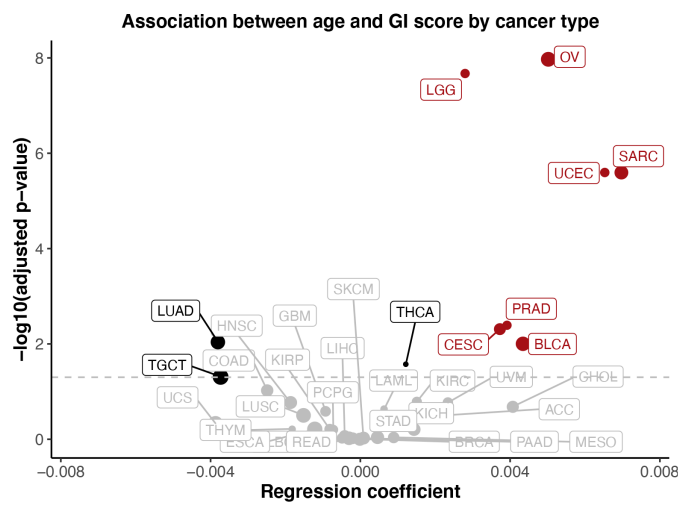

d

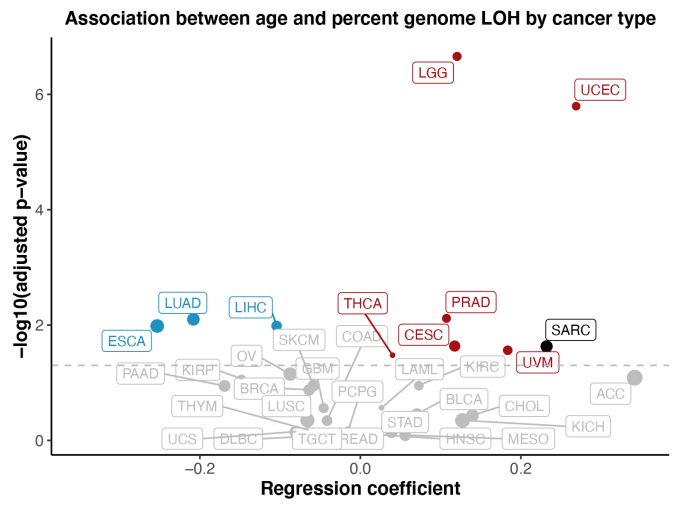

ov

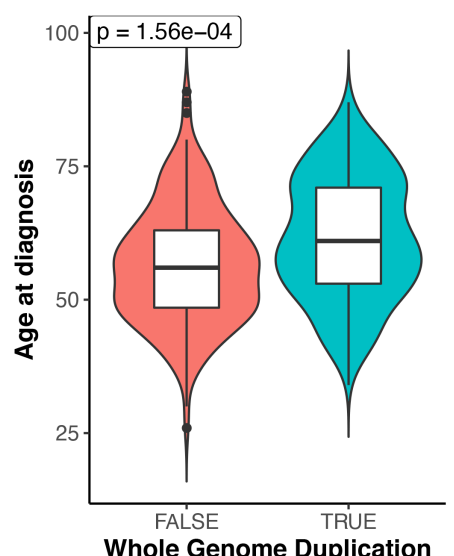

UCEC

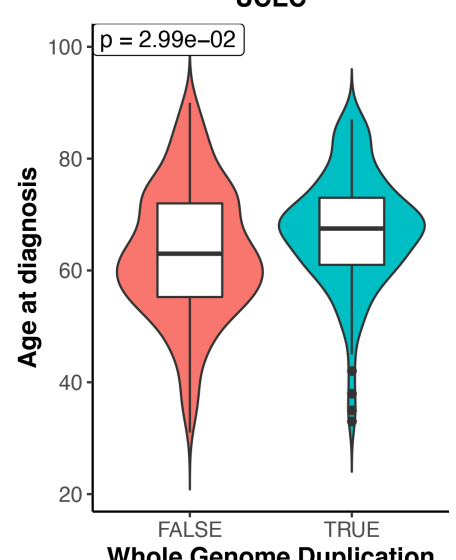

Figure 1 
bioRxiv preprint doi: https://doi.org/10.1101/2020.08.25.266403; this version posted August 25, 2020. The copyright holder for this preprint (which was not certified by peer review) is the author/funder, who has granted bioRxiv a license to display the preprint in perpetuity. It is made available under aCC-BY 4.0 International license.

a

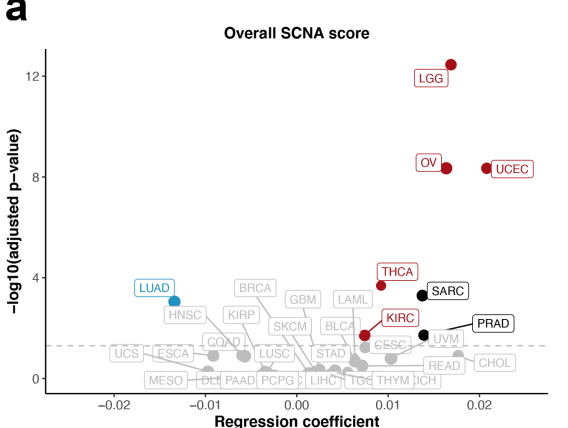

b

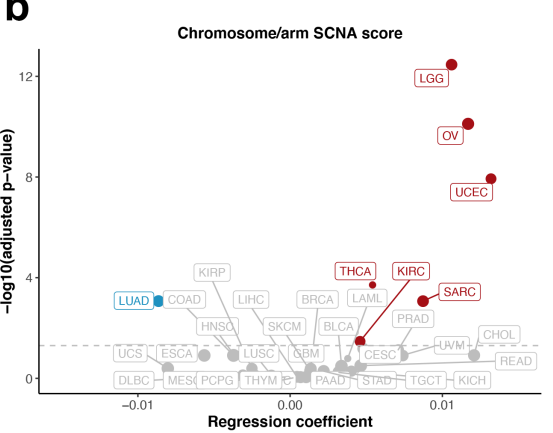

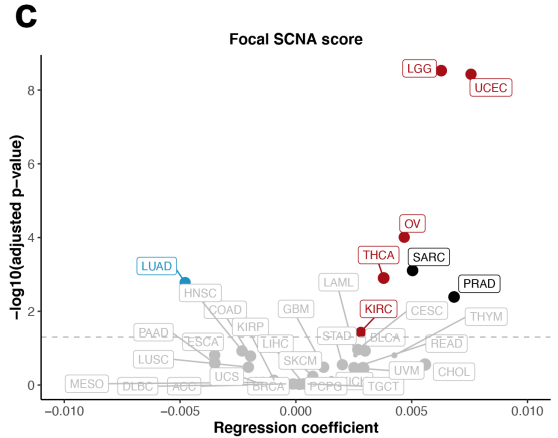

d

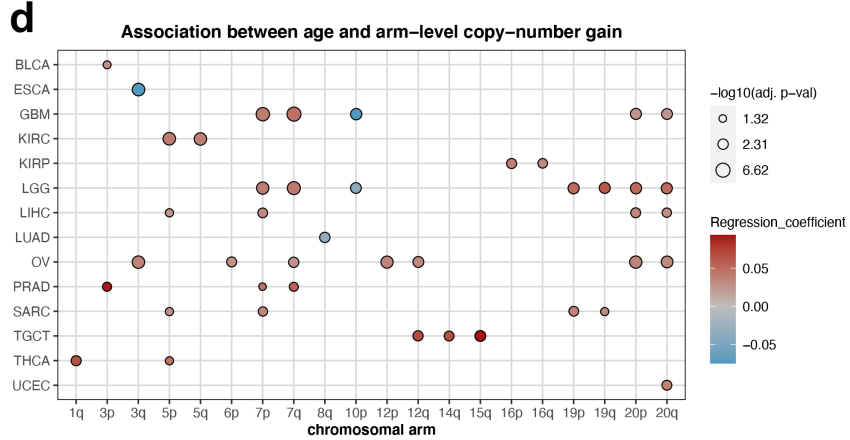

f

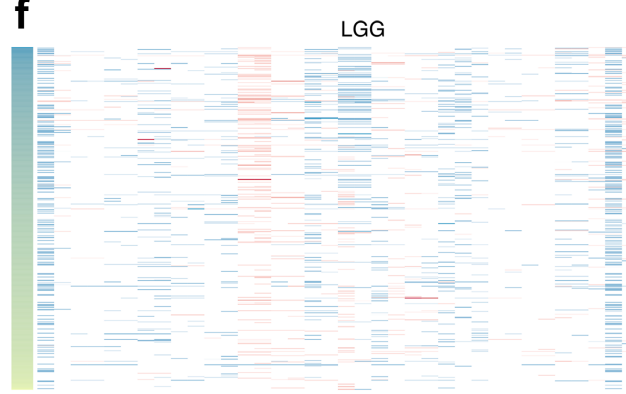

\% e Association between age and arm-level copy-number loss

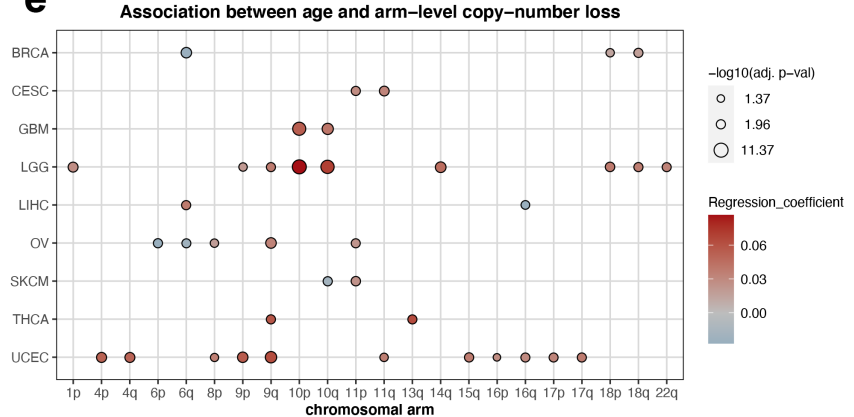

g

UCEC

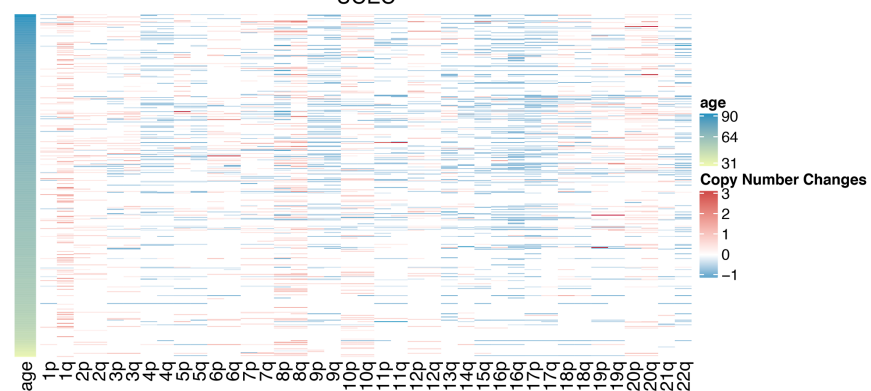

Figure 2 
bioRxiv preprint doi: https://doi. org/10.1101/2020 08 25.266403; this version posted August 25, 2020. The copyright holder for this preprint (which was not certified by peer review) is the author/funder, who has granted bioRxiv a license to display the preprint in perpetuity. It is made available under aCC-BY 4.0 International license.

a

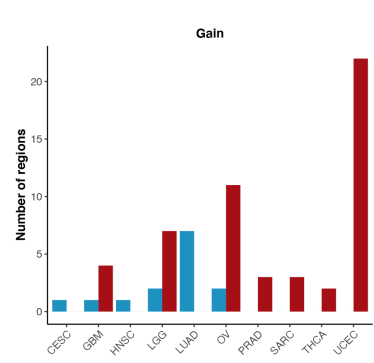

b

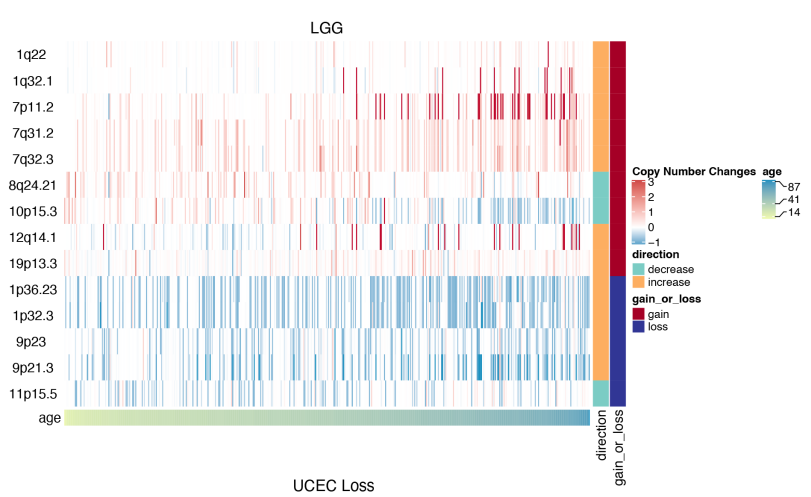

C
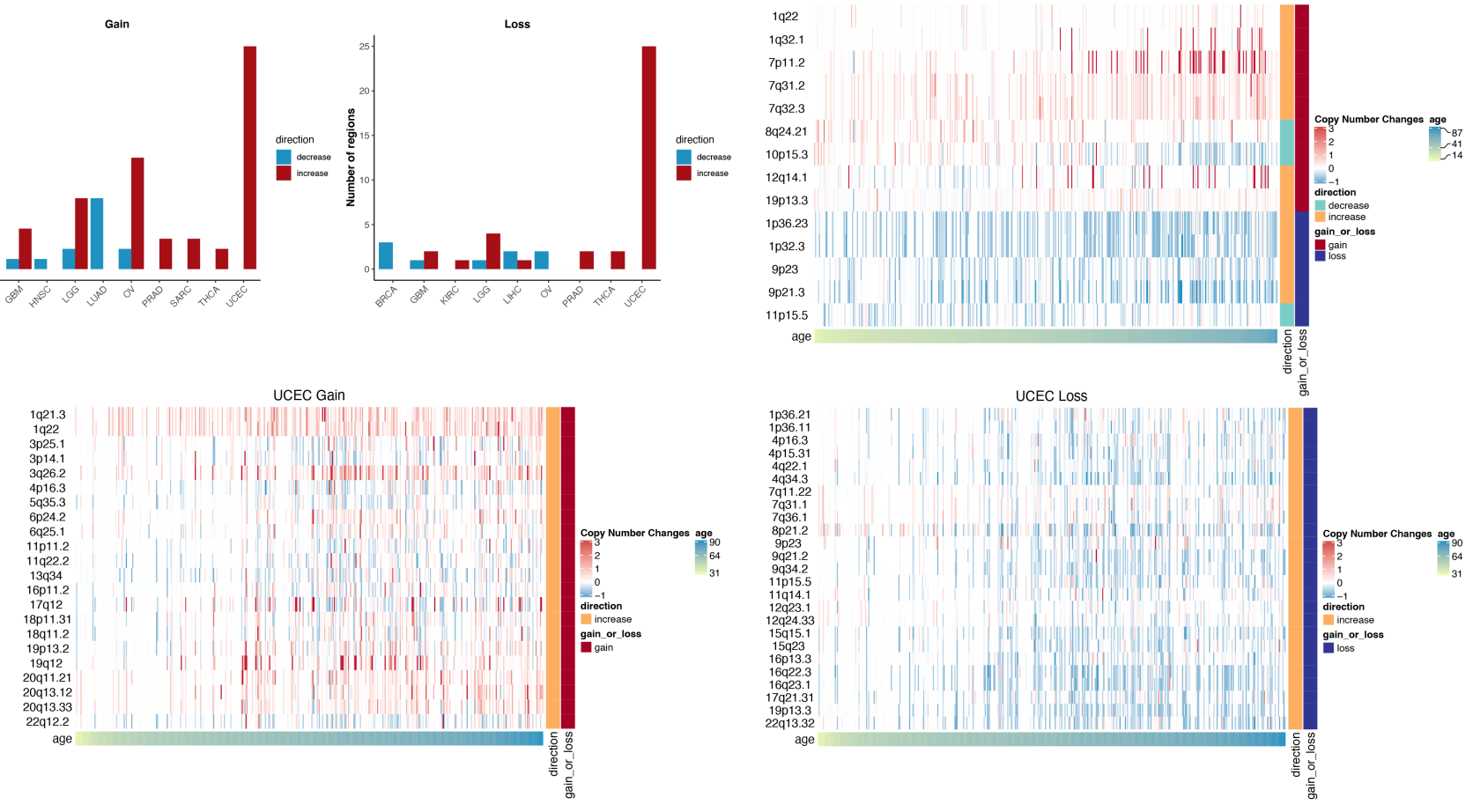

$$
\begin{aligned}
& 1 \mathrm{q} 2 \\
& 1 \mathrm{q} 3 \\
& 7 \mathrm{p} 1 \\
& 7 \mathrm{q} 3 \\
& 7 \mathrm{q} 3 \\
& 8 \mathrm{q} 2 \\
& 10 \mathrm{p} \\
& 12 \mathrm{q} \\
& 19 \mathrm{p} \\
& 1 \mathrm{p} 3 \\
& 1 \mathrm{p} 3 \\
& 9 \mathrm{p} 2 \\
& 9 \mathrm{p} 2 \\
& 11 \mathrm{p} \\
& \hline
\end{aligned}
$$

d

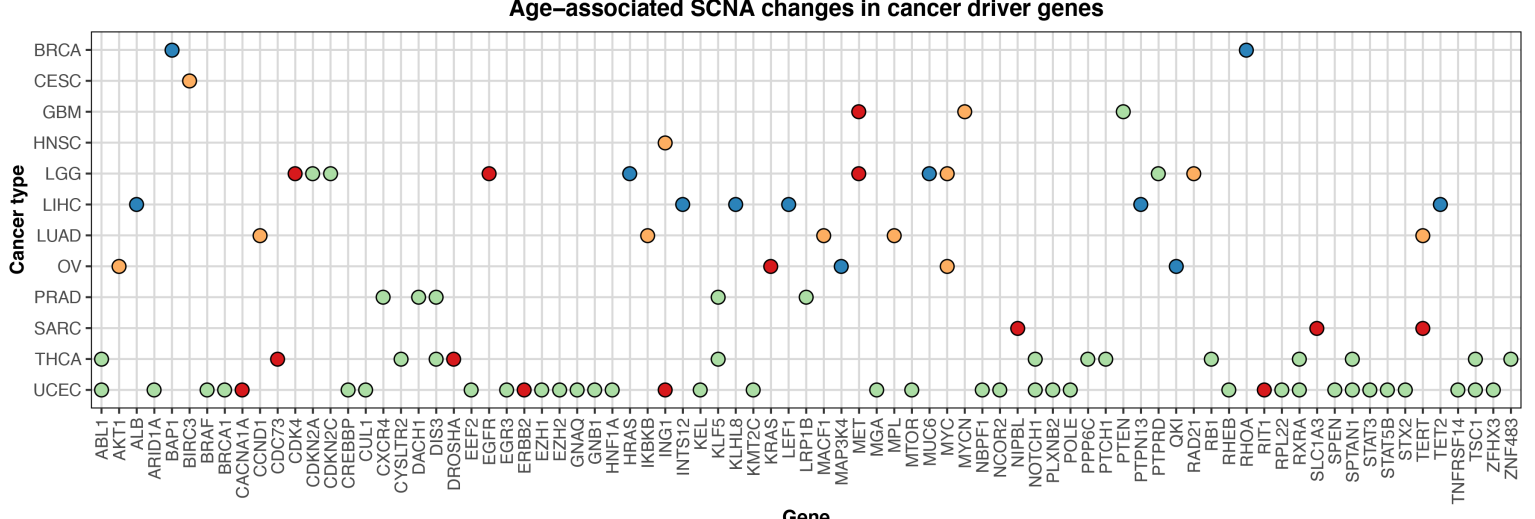

Decrease gain

- Increase gain

- Decrease loss

Increase loss

e
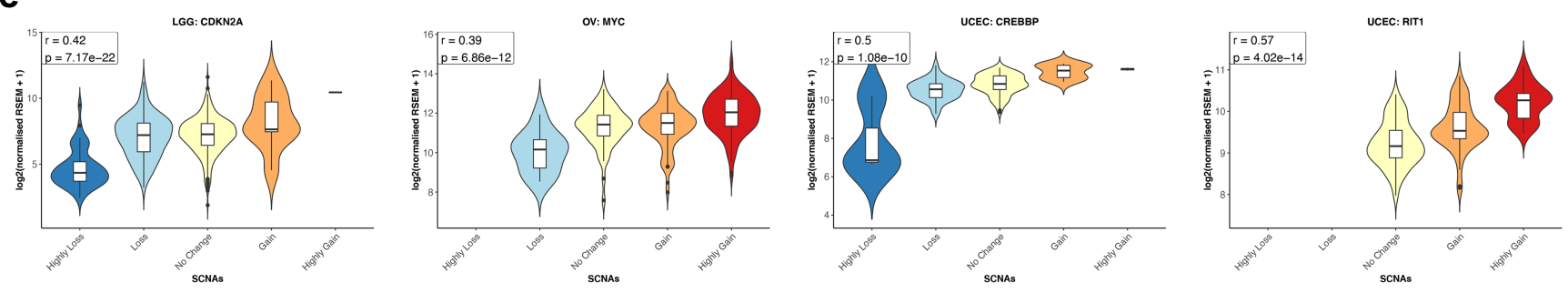

Figure 3 
bioRxiv preprint doi: https://doi.org/10.1101/2020.08 25.266403; this version posted August 25, 2020. The copyright holder for this preprint (which was not certified by peer review) is the author/funder, who has granted bioRxiv a license to display the preprint in perpetuity. It is made available under aCC-BY 4.0 International license.

a

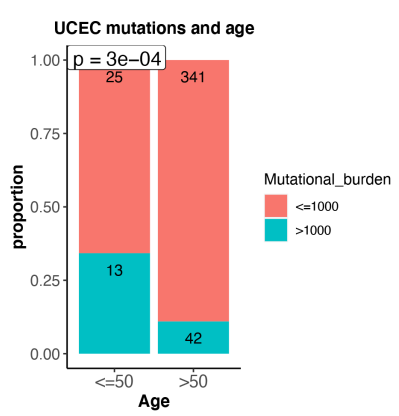

b

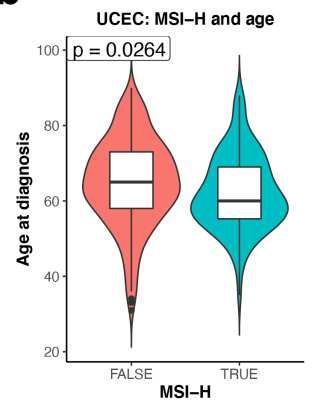

C

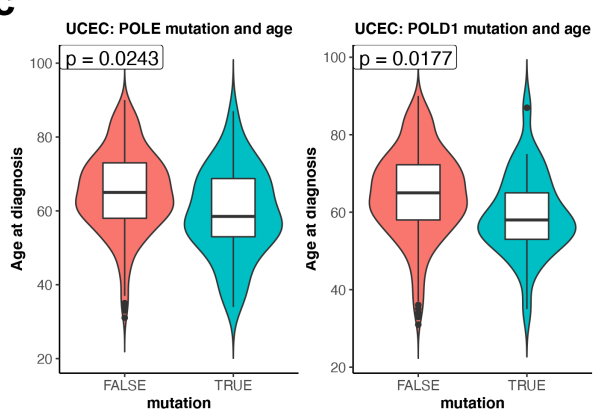

d

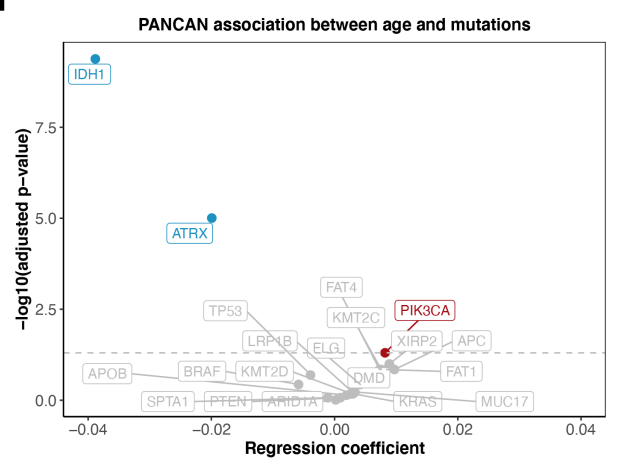

e

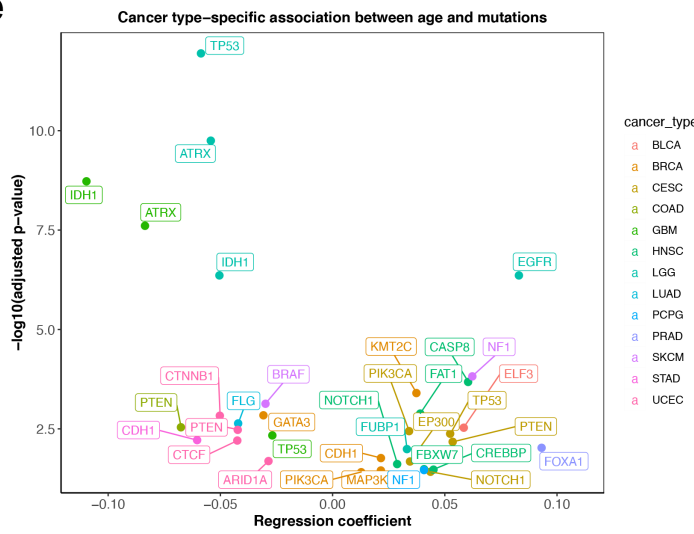

f

GBM

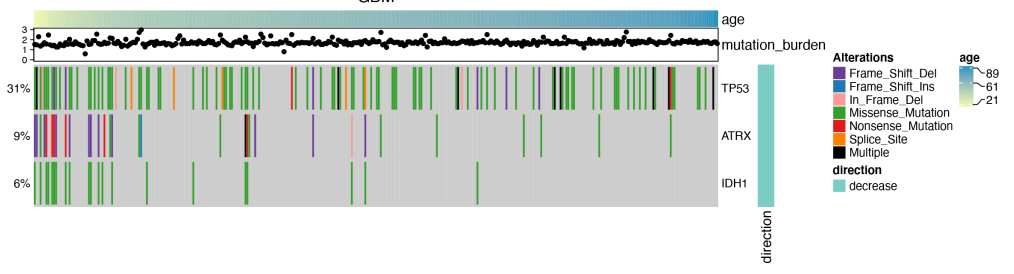

LGG

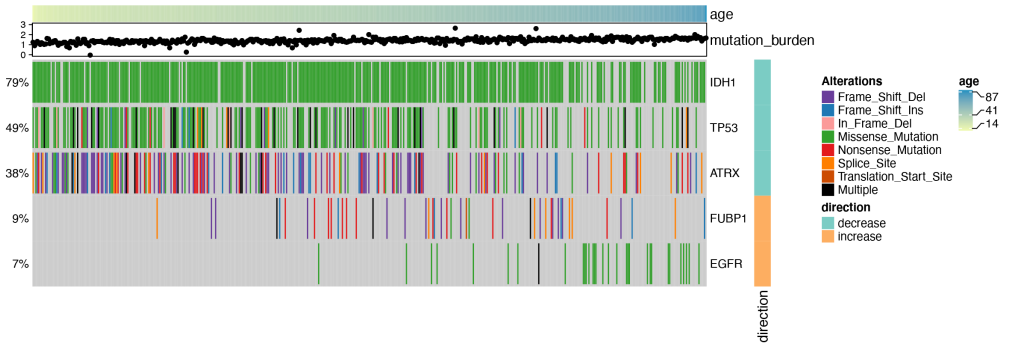

Figure 4 
bioRxiv preprint doi: https://doi.org/10.1101/2020.08 25.266403; this version posted August 25, 2020. The copyright holder for this preprint (which was not certified by peer review) is the author/funder, who has granted bioRxiv a license to display the preprint in perpetuity. It is made available under aCC-BY 4.0 International license.

a

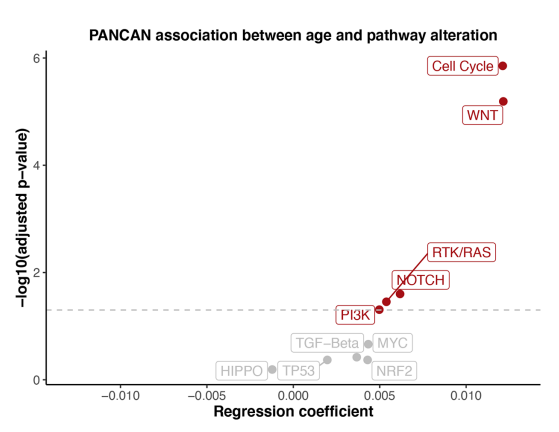

b

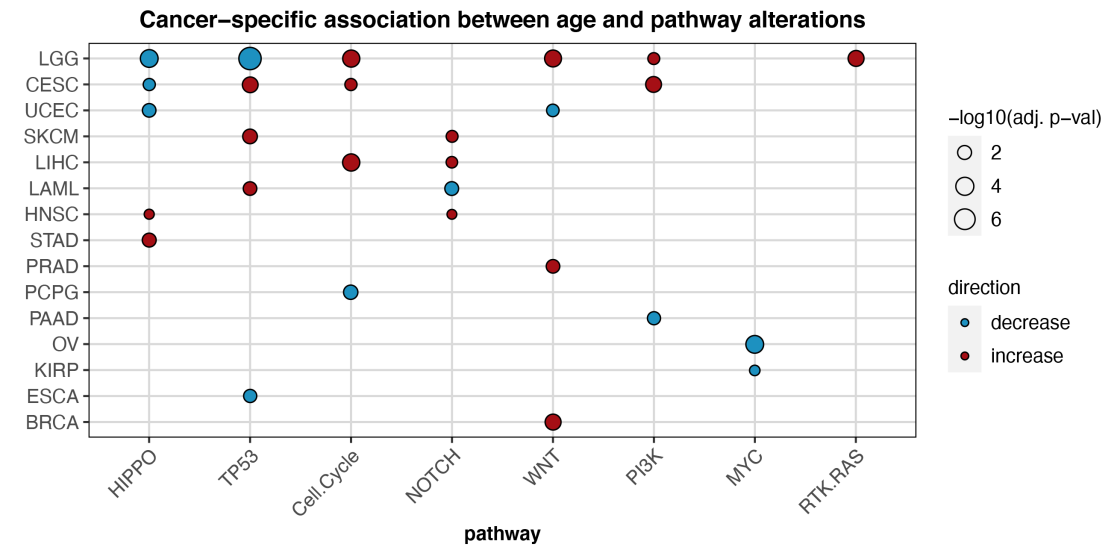

C

LGG: TP53

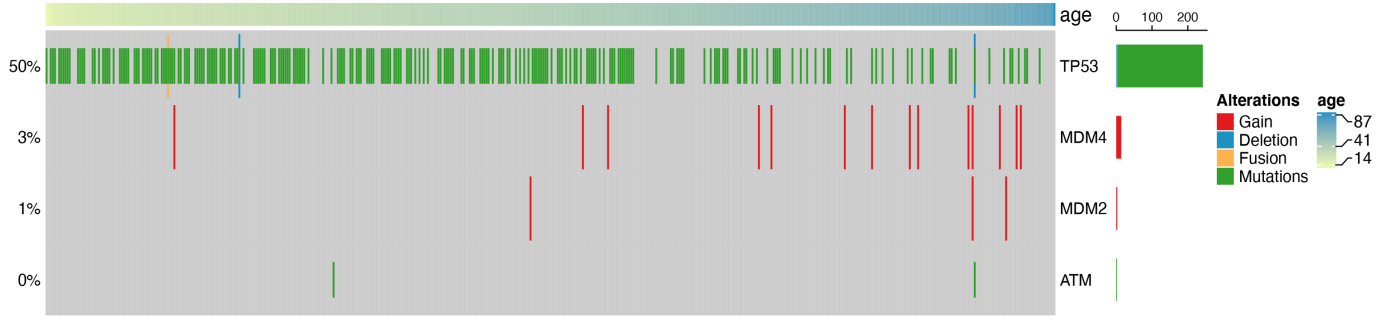

LGG: Cell Cycle

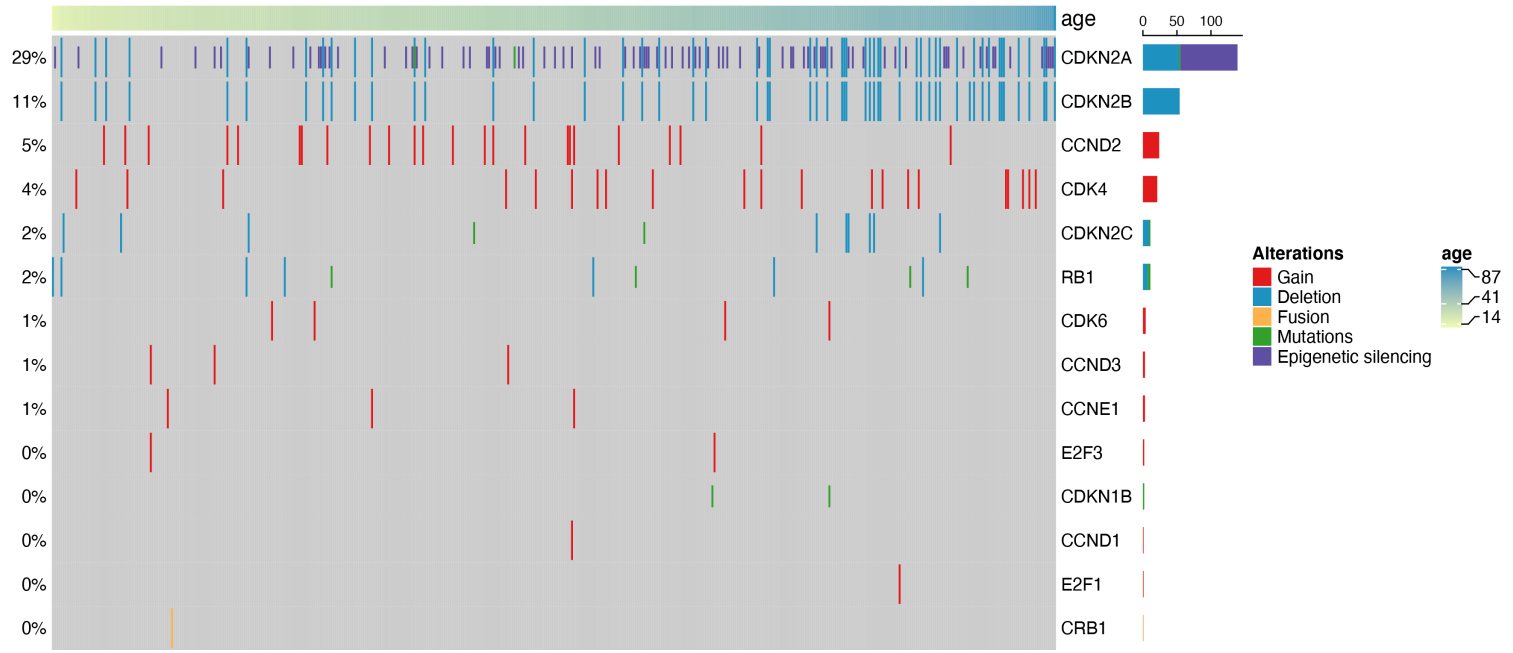

Figure 5 
bioRxiv preprint doi: https://doi.org/10.1101/2020.08 25.266403; this version posted August 25, 2020. The copyright holder for this preprint (which was not certified by peer review) is the author/funder, who has granted bioRxiv a license to display the preprint in perpetuity. It is made available under aCC-BY 4.0 International license.

a

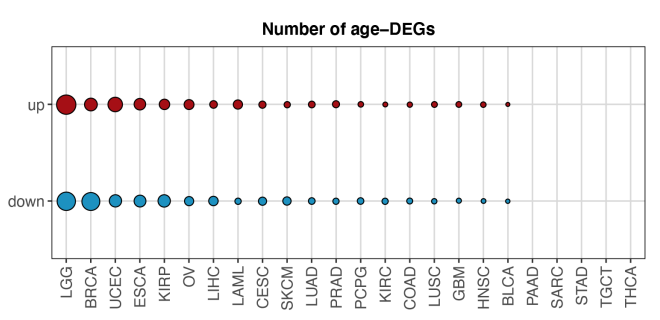

direction
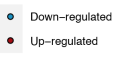

number_of_genes

$\circ 50$

$\begin{array}{ll} & 500 \\ & 1000\end{array}$

2000
2500

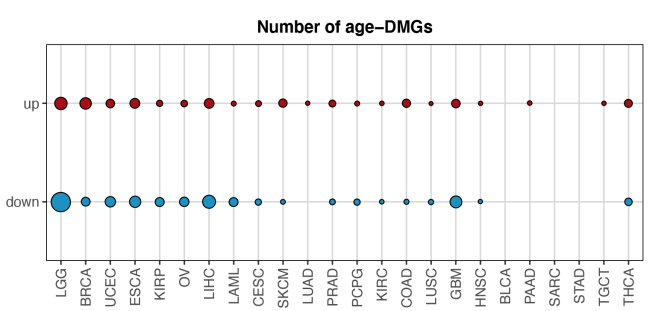

direction

- Down-regulated - Up-regulated

number_of_genes

- 100

○ 500

○ 1000

$\begin{array}{ll}\bigcirc & 2000 \\ \bigcirc & 3000\end{array}$

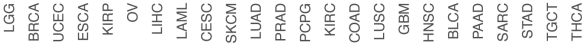

b

LGG

BRCA

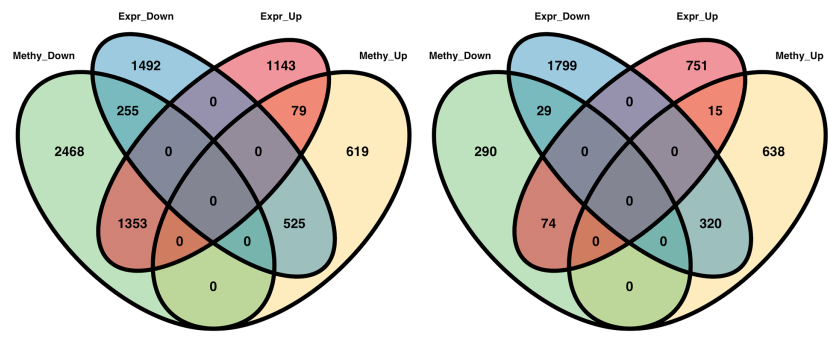

C

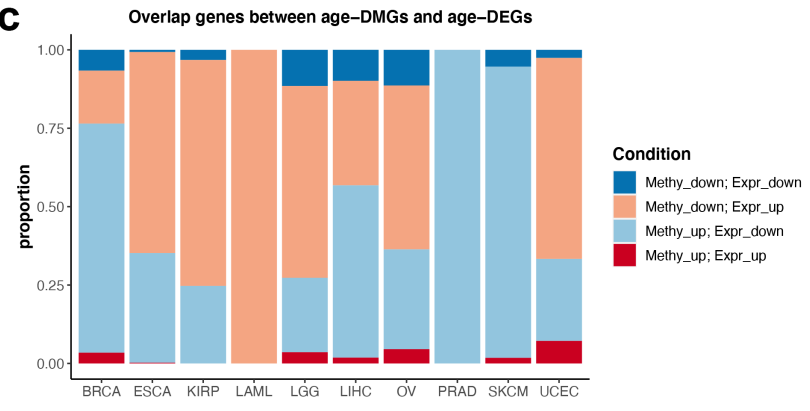

d

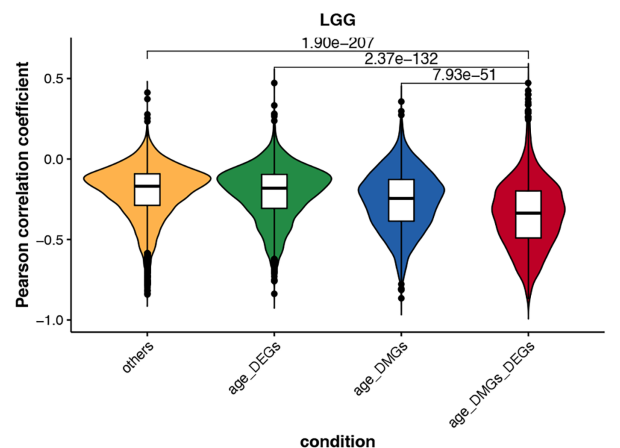

e

GSEA: LGG

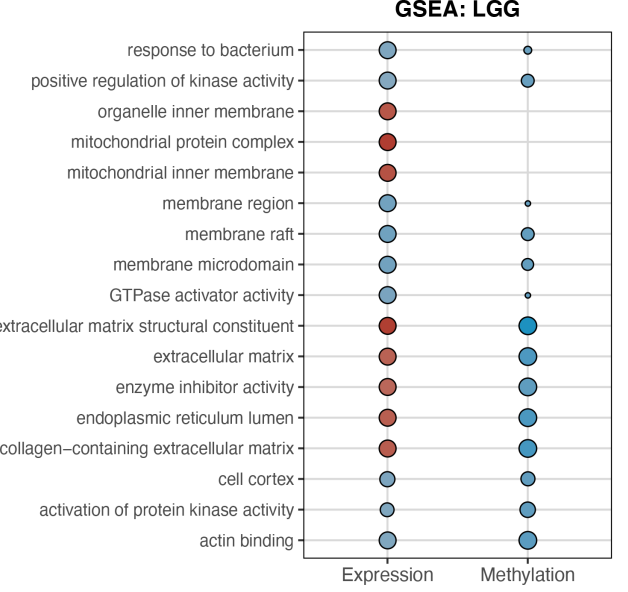

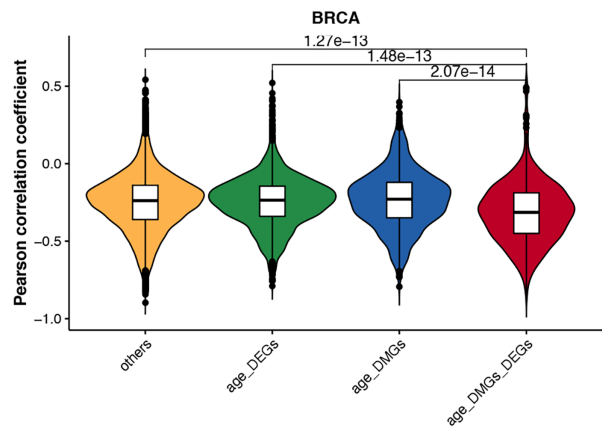

condition

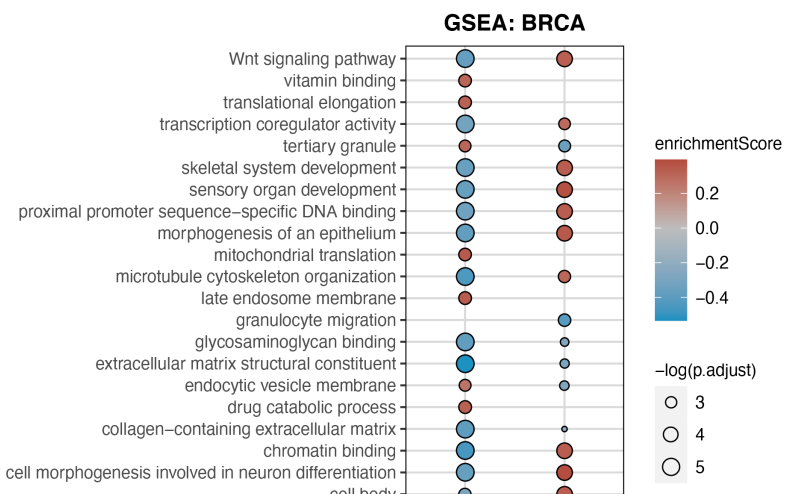

Figure 6 